\title{
Special Cases during the Detection of the Hook Style Energy Theft in Overhead Low-Voltage Power Grids through HS-DET Method - Part 1: High Measurement Differences, Very Long Hook Technique and "Smart" Hooks
}

\author{
Athanasios G. Lazaropoulos* \\ School of Electrical and Computer Engineering / National Technical University of Athens / \\ 9 Iroon Polytechniou Street / Zografou, GR 15780
}

Received November 01, 2018; Accepted December 18, 2018; Published December 26, 2018

\begin{abstract}
On the basis of [1], this pair of companion papers investigates the possibility of jamming the method of the detection of the hook style energy theft (HS-DET method) that can be used for the detection of the hook style energy theft in the overhead low-voltage (OV LV) power grids. The three main suspicious issues that have been identified in [1] are further investigated in this paper. The robustness of the HS-DET method against these issues is assessed by using percent error sum (PES) submetrics, appropriate contour plots and a new proposed robustness PES submetric against the hook style energy theft of HS-DET method.
\end{abstract}

Keywords: Smart Grid; Broadband over Power Lines (BPL); Power Line Communications (PLC); Distribution Power Grid; Energy Theft; Jamming; Robustness

\section{Introduction}

A portfolio of Broadband over Power Lines (BPL) applications, such as Topology Identification Methodology (TIM) [2], Fault and Instability Identification Methodology (FIIM) [3], methodology to preserve power system stability [4], [5] and main line fault localization methodology (MLFLM) [6]-[8], have already been discussed. In earlier published literature [1], the hook style energy theft detection method (HS-DET method) has been proposed and added in the aforementioned broadband application portfolio thus contributing towards a more accurate and more reliable monitoring, metering and controlling of distribution power grids.

HS-DET method is based on the hybrid model [9]-[26] while HS-DET method output is expressed in terms of appropriate percent error sum (PES) submetrics and contour plots. As the hybrid model is concerned, it consists of two interconnected modules, namely: (i) the bottom-up approach module; and (ii) the top-down approach module. The channel attenuation, that is one of the provided outputs of the hybrid method for given OV LV BPL topology and BPL operation frequency range, is further processed by the HS-DET method so that PES metrics can be produced. Depending on the values of the PES metrics, the decision regarding the existence of hook style energy theft in 
overhead low-voltage (OV LV) BPL networks can be made. On the basis of PES metrics, the impact of different hook characteristics, OV LV BPL topologies and different intensities of measurement differences can be expressed in terms of appropriate contour plots [1].

In accordance with [1] and if all the other problematic cases, which concern the operation of the OV LV power grid and described in [2]-[8], are excluded, HS-DET method can safely detect hook style energy thefts that may occur across the OV LV BPL networks. As indicated in [1], safe decisions concerning the existence of energy theft or not can be made regardless of the examined OV LV BPL topology, the intensity of the measurement differences and the hook characteristics. However, three critical issues have been recognized during the numerical result section of [1] that may jam HS-DET method and need further examination in order to assess the extent of jamming that may create. With reference to [1], these three special cases are: (i) the existence / definition of the threshold of the intensity of measurement differences that may occur / be produced by external sources above which HS-DET method starts to give faulty decisions concerning the existence of hook style energy theft; (ii) the possibility of the installation of very long hooks in order to mask the hook existence during the application of HS-DET method, which is anyway a broadband technique; and (iii) the use of "smart" hooks that are matched to the characteristic impedances of the lines in order again to cover the energy theft. In the following sections, the mitigation efficiency of HS-DET against these three special cases will be assessed either in theoretical or in numerical terms via theoretical analysis, PES metrics and contour plots.

The rest of this paper is organized as follows: In Section 2, a brief synopsis of HS-DET method and its PES metrics is given. Also, the theoretical basis is presented in order to cope with the three examined special cases. In Section 3, numerical results and discussion are provided, aiming at practically evaluating the extent of jamming to HS-DET method that may create the three examined special cases as well as the possibility of masking the hook style energy theft exists. Section 4 concludes this paper.

\section{HS-DET Method Synopsis}

\subsection{Measurement Differences}

As already been mentioned in [1], the measurement differences that are used during the BPL simulations describe the significant differences that may occur between experimental measurements and theoretical results. Anyway, the mathematical consideration of the measurement differences remains a well-defined straightforward process, since the total measurement difference can be conveniently assumed to follow continuous uniform distribution (CUD) with minimum value $-a_{\text {CUD }}$ and maximum value $a_{\text {CUD }}[2],[6]-[8],[27]$. On the basis of the CUD measurement difference $e\{\cdot\}$, the measured coupling scheme transfer function $\overline{H^{\mathrm{C}}}\{\cdot\}$ for given coupling scheme can be determined by

$$
\overline{H^{\mathrm{C}}}\left(f_{i}\right)=H^{\mathrm{C}}\left(f_{i}\right)+e\left(f_{i}\right), i=1, \ldots, u
$$

where $H^{\mathrm{C}}\{\cdot\}$ is the theoretical coupling scheme transfer function, $f_{i}, i=1, \ldots, u$ denotes the measurement frequency, $u$ is the number of subchannels in the examined frequency range and $e\left(f_{i}\right)$ synopsizes the total measurement difference in $\mathrm{dB}$ at frequency $f_{i}$. Note that the theoretical coupling scheme transfer function is among the outputs of the hybrid method 
and depends on the examined MTL configuration, OV LV BPL topology and the applied coupling scheme [9], [12], [15], [17]-[19], [28], [29].

\subsection{Impact of the Hook Insertion}

With reference to Fig. 2(b) of [1], each hook is characterized by: (i) its length $L_{\mathrm{bh}}$; and (ii) its distance $D_{\mathrm{h}}=L_{\mathrm{h}}+\sum_{i=1}^{k-1}\left(L_{i}\right)$ from the transmitting end. To facilitate the application of the hybrid method, the hook termination is assumed to be: (a) open circuit; (b) fully interconnected with the distribution lines (i.e., hook derivation points at the same distance from the transmitting end on all the three phases); and (c) horizontal. The aforementioned three assumptions concerning the hook insertion permit its handling as branch from the hybrid method.

Apart from the hook characteristics and hook assumptions, a set of settings regarding the OV LV BPL topology operation should be taken under consideration as usually. More specifically, the topological characteristics of the indicative OV LV BPL topologies of Table 1 of [1] as well as a set of assumptions concerning the transmission and propagation of the BPL signal and the circuital parameters that are detailed in [9], [10]-[17], [19], [21], [30]-[34] are again assumed in this paper.

Obviously, the hook insertion modifies the original OV LV BPL topology to the respective modified one thus influencing the determination of coupling scheme transfer functions. With reference to eq. (1) and [1], $H_{\mathrm{nr}}^{\mathrm{C}}\{\cdot\}$ and $H_{\bmod }^{\mathrm{C}}\{\cdot\}$ are the original and modified theoretical coupling scheme channel transfer functions, respectively, while $\overline{H_{\mathrm{nr}}^{\mathrm{C}}}\{\cdot\}$ and $\overline{H_{\text {mad }}^{\mathrm{C}}}\{\cdot\}$ are the original and modified measured coupling scheme channel transfer functions, respectively. Note that it is assumed that the measured coupling scheme transfer function of original and modified end-to-end OV LV BPL topologies suffers from the same total measurement differences.

\subsection{PES Submetrics of HS-DET Method and Special Cases}

PES submetrics define the cornerstone metrics of HS-DET method. However, PES submetrics are not the first time that are used by BPL applications since they have already been used in TIM [2], FIIM [3], power system stability methodology [4], [5] and MLFLM [6]-[8] in order to assess the approximation accuracy of the original and modified coupling scheme transfer functions. With reference to eq. (1) and Sec. 2.2, the PES submetrics that are used in this paper are:

$$
\begin{gathered}
\overline{P E S_{\text {or }}}=100 \% \cdot \frac{\sum_{i=1}^{u}\left|\overline{H_{\mathrm{or}}^{C}}\left(f_{i}\right)-H_{\mathrm{or}}^{\mathrm{C}}\left(f_{i}\right)\right|}{\sum_{i=1}^{u}\left|H_{\mathrm{or}}^{\mathrm{C}}\left(f_{i}\right)\right|} \\
\overline{P E S_{\bmod }}=100 \% \cdot \frac{\sum_{i=1}^{u} \mid \frac{H_{\mathrm{mod}}^{\mathrm{C}}}{\left.\mid f_{i}\right)-H_{\mathrm{or}}^{\mathrm{C}}\left(f_{i}\right) \mid}}{\sum_{i=1}^{u}\left|H_{\mathrm{or}}^{\mathrm{C}}\left(f_{i}\right)\right|}
\end{gathered}
$$

where $\overline{P E S_{\mathrm{rr}}}$ and $\overline{P E S_{\text {mod }}}$ assess the accuracy of the original measured coupling scheme channel transfer function and modified measured coupling scheme channel transfer functions, respectively, in relation with the original theoretical coupling scheme channel transfer function. With reference to eqs. (2) and (3), the main PES submetric of HS-DET method is the difference between the original measured coupling scheme channel transfer function and modified measured coupling scheme channel transfer functions, say

$$
\overline{\triangle P E S}=\overline{P E S_{\text {mad }}}-\overline{P E S_{\mathrm{nr}}}
$$

On the basis of $\overline{\triangle P E S}$, appropriate contour plots can be plotted so that the detection ability of the HS-DET method can be assessed with respect to the hook length and the distance from the transmitting end [1]. 
In accordance with [1] and if all the other problematic cases, which concern the operation of the OV LV power grid and described in [2]-[8], are excluded, HS-DET method can safely detect hook style energy thefts through $\overline{\triangle P E S}$. Actually, the definition of $\overline{\triangle P E S}$ implies that the larger part of measurement differences can be mutually mitigated. Since the role of measurement differences is typically eliminated, values of $\overline{\triangle P E S}$ above zero imply that modified measured coupling scheme channel transfer function is more different than the original measured coupling scheme channel transfer function with respect to the original theoretical coupling scheme channel transfer function. Greater values of $\overline{\triangle P E S}$ mean greater differences between the modified and the original OV LV BPL topology and, thus, a safer decision regarding the existence of an energy theft can be secured.

Although the vast majority of the serious problematic cases can be detected through the aforementioned set of BPL broadband applications, small differences can occur during the computation of the coupling transfer functions due to secondary reasons such as temperature, wind, humidity, etc. These small differences can cause false energy theft alarm. Hence, a threshold for $\overline{\triangle P E S}$ should be assumed so that the likelihood of false energy theft alarm due to secondary reasons can be reduced without jeopardizing the performance of HS-DET method. Since the measurement differences can be satisfactorily mitigated due to the definition of $\overline{\triangle P E S}$, a fixed percentage for all the examined OV LV BPL topologies can act as a proper $\overline{\triangle P E S}$ threshold (see Sec.3).

Although the theoretical framework of HS-DET method is well-defined and the assessment of the previous findings have been made in [1], three special cases have been identified that may foment the operation of HS-DET method. More specifically:

- High measurement differences that may intentionally / unintentionally be produced can jam $H S-D E T$ method. As already been mentioned, due to the definition of $\overline{\triangle P E S}, \overline{\triangle P E S}$ is almost invulnerable to the measurement differences since the larger part of them has already been mitigated. Hence, it is expected that even if high maximum values $a_{\text {CUD }}$ are adopted, HS-DET method can still detect the energy theft through the positive values of $\overline{\triangle P E S}$ (but even safer above the $\overline{\triangle P E S}$ threshold). However, the robustness of the decision, that mainly depends on the intensity of measurement differences, should be computed; say, the degree of certainty. Robustness of the decision concerning the existence of energy theft is given by

$$
\overline{R o b}=100 \% \cdot \frac{\overline{\triangle P E S}}{\overline{P E S_{\mathrm{nr}}}}
$$

This additional PES submetric that assesses the quality of the decision concerning the existence of energy theft demands high values. High values of $\overline{R o b}$ allow high margins of certainty.

- The installation of very long hooks in order to mask the hook existence during the application of HS-DET method. By misinterpreting the trends of Figs. 3-8 of [1], it can be mentioned that since $\overline{\triangle P E S}$ negatively depends on the hook length, very long hooks could jam HS-DET method. However, this assertion is not valid since MTL configurations such as of OV LV BPL topologies are analyzed on the basis of TL theory. This is going to be validated in Sec. 3.2. 


\section{Numerical Results and Discussion}

The numerical results of this Section focus on assessing the performance of HSDET method to successfully detect the potential energy theft when high measurement differences, which may intentionally / unintentionally be produced, and the installation of very long hooks or "smart" hooks occur. All these three special cases try to jam HS-DET method. For that reason, the robustness of decisions of HS-DET method is also evaluated.

\subsection{High Measurement Differences and HS-DET Method Jamming}

The influence of measurement differences of maximum value $a_{\text {CUD }}$ up to $5 \mathrm{~dB}$ has been thoroughly discussed in [1] while the influence of measurement differences of maximum value $a_{\text {CUD }}$ above $5 \mathrm{~dB}$ is here evaluated. For maximum values $a_{\text {CuD }}$ below 5 $\mathrm{dB}$, it has been verified that the stochastic nature of measurement differences mainly affects OV LV BPL topologies with branches. Also, on the basis of $\overline{\triangle P E S}$ contour plots, the increase of the CUD maximum value of measurement differences negatively influences the performance of HS-DET method since a general reduction of $\overline{\triangle P E S}$ values of contour plots is observed with the increase of maximum value $a_{\text {cuD }}$. However, HSDET method successfully detected the energy theft in all the cases examined regardless of the CUD maximum value of measurement differences since $\overline{\triangle P E S}$ values have been always greater than $0 \%$. In this paper, the influence of high measurement differences (i.e., measurement differences of maximum value $a_{\text {cuD }}$ above $5 \mathrm{~dB}$ ) is evaluated in this paper. In accordance with Sec. 2.3, the performance of HS-DET method against high measurement differences will be assessed in terms of $\overline{\triangle P E S}, \overline{P E S_{\mathrm{rr}}}$ and $\overline{R o b}$.

With reference to Fig. 2(b) of [1] and the indicative original OV LV BPL topologies as reported in Table 1 of [1], let assume that a hook of length $L_{\text {bh }}$ is inserted at distance $D_{\mathrm{h}}$ from the transmitting end. In Fig. 1(a), $\overline{\triangle P E S}$ is plotted with respect to the maximum value $a_{\text {CUD }}$ when $L_{\mathrm{bh}}=5 \mathrm{~m}$ and $D_{\mathrm{h}}=300 \mathrm{~m}$ are assumed for the five indicative original OV LV BPL topologies. In Figs. 1(b) and 1(c), same curves with Fig. 1(a) are given but for $\overline{P E S_{\mathrm{nr}}}$ and $\overline{R o b}$, respectively. 


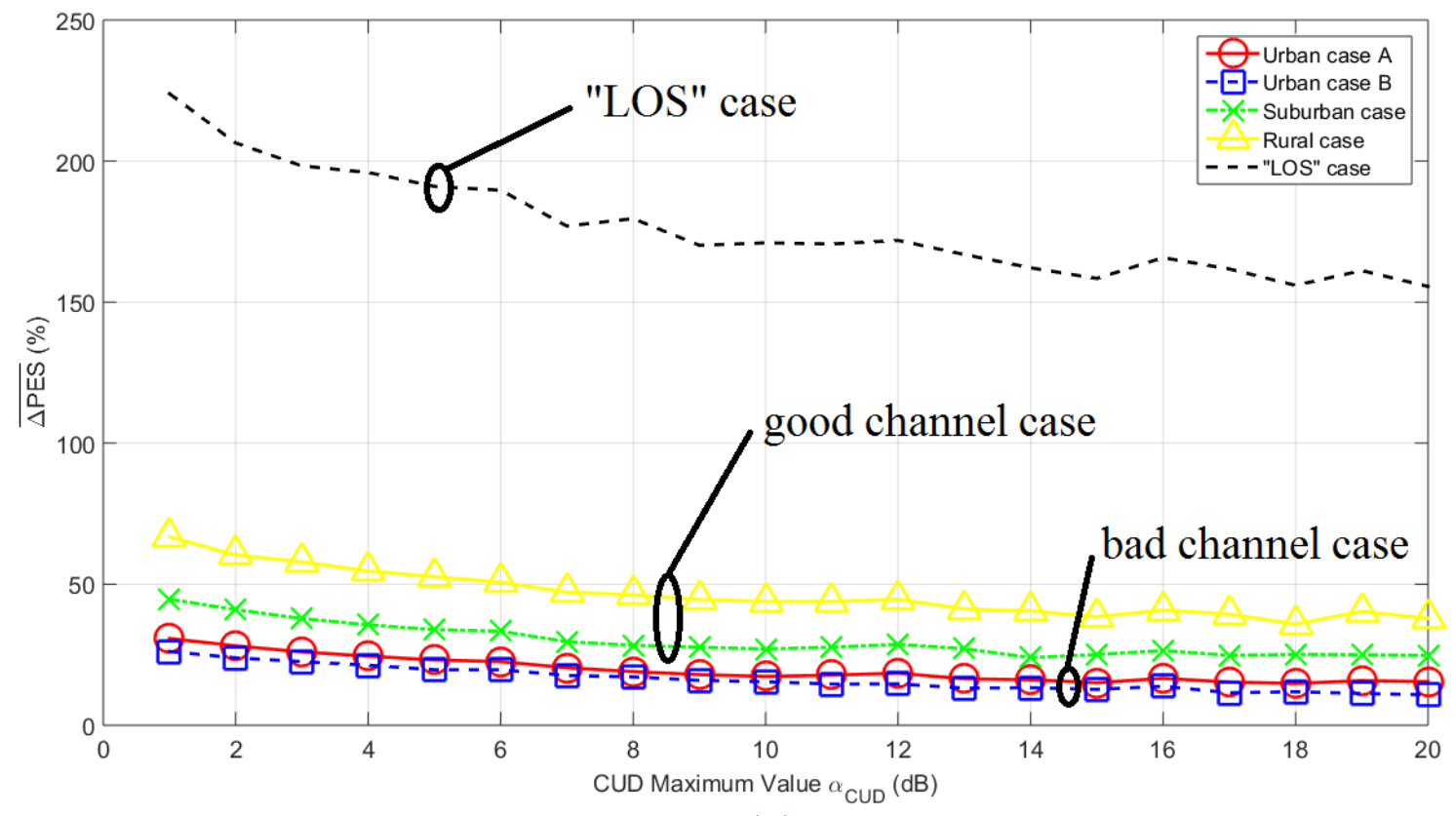

(a)

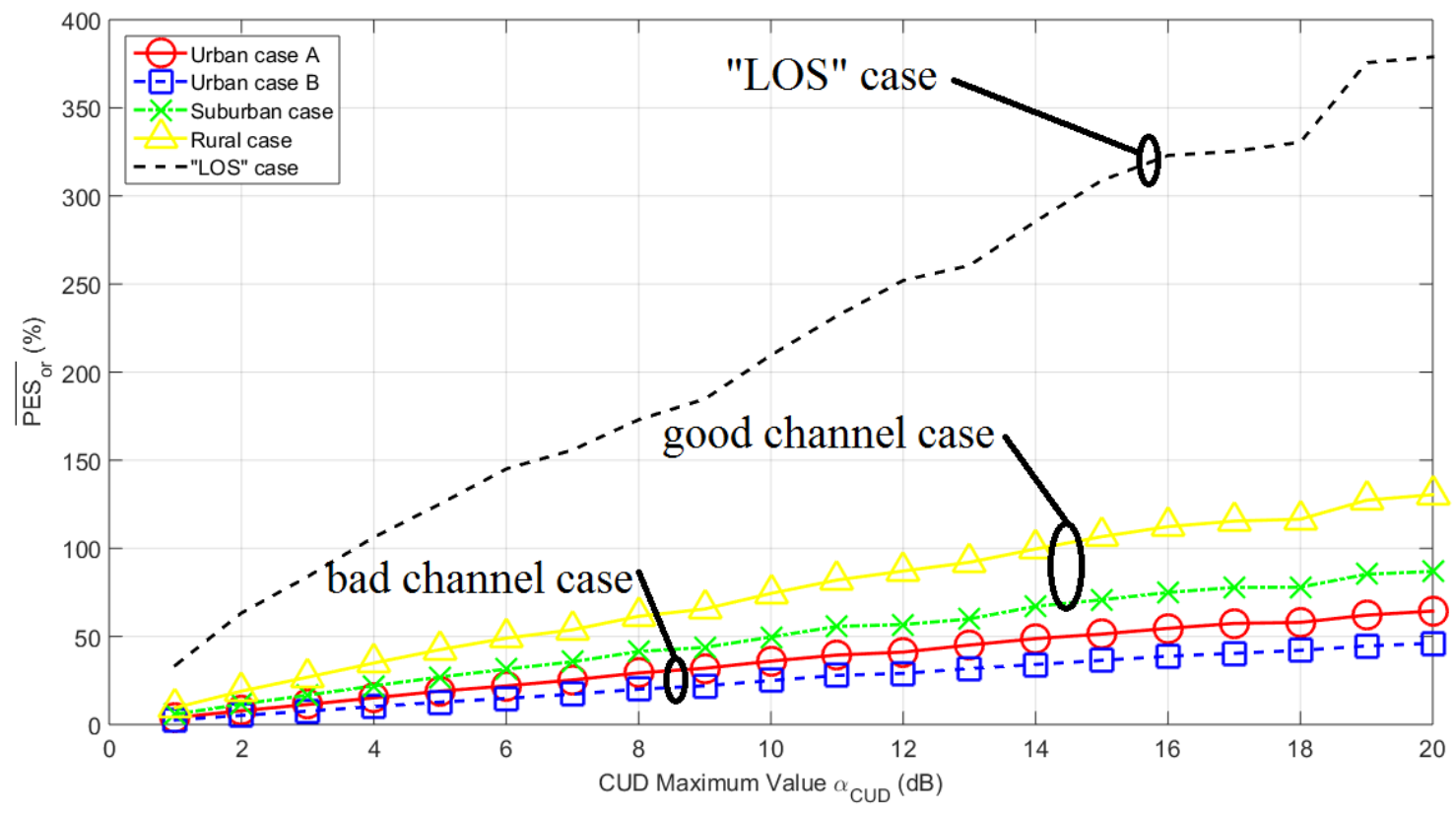

(b) 


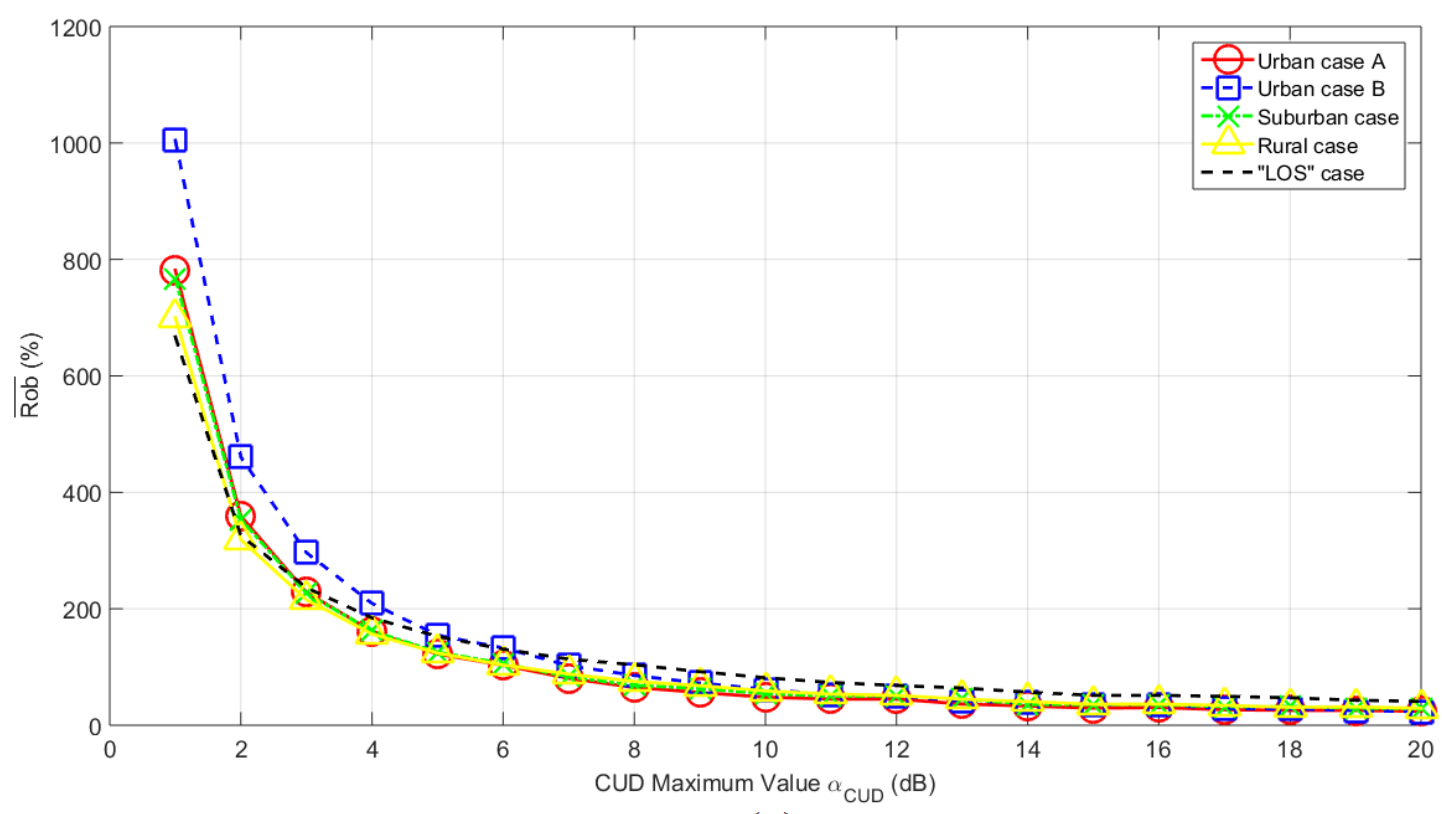

(c)

Fig. 1. PES submetrics of HS-DET method for the five original indicative OV LV BPL topologies of [1] when hook length of $5 \mathrm{~m}$, hook distance from the transmitting end of $300 \mathrm{~m}$ and open-circuit hook termination are assumed for various maximum values $a_{\text {CUD }}$. (a) $\overline{\triangle P E S}$. (b) $\overline{P E S_{\text {or }}}$. (c) $\overline{R o b}$.

From Figs. 1(a) and 1(b), it is evident that $\overline{\triangle P E S}$ and $\overline{P E S_{\mathrm{n}}}$ present similar results that clarify the impact of high measurement differences on the HS-DET method. Actually, three categories of OV LV BPL topologies can be defined, say:

- "LOS" case: Even if measurement differences of high maximum value $a_{\text {cud }}$ are considered, their impact on the performance of HS-DET method is anemic. In all the cases examined, $\overline{\triangle P E S}$ remains over $150 \%$ thus indicating the easy detection of energy theft in OV LV BPL "LOS" case. Although $\overline{P E S_{\mathrm{rr}}}$ of "LOS" case presents the highest values in comparison with the respective values of the other examined modified OV LV BPL topologies, HS-DET method can successfully mitigate the measurement differences and give a secure decision concerning the existence of energy theft.

- Good channel case: Good channel case consists of the suburban and rural modified OV LV BPL topologies. Although $\overline{\triangle P E S}$ is significantly lower than the respective value of the "LOS" case, its value remains high enough to secure a decision concerning the existence of energy theft regardless of the maximum value $a_{\mathrm{CUD}}$.

- Bad channel case: On the basis of $\overline{\triangle P E S}$ of urban case $\mathrm{A}$ and $\mathrm{B}$, this is the most difficult decision concerning the existence of power theft. $\overline{\triangle P E S}$ receives low values but always positive. It is obvious that an arbitrary $\overline{\triangle P E S}$ threshold concerning the strict decision about the existence of power theft can be estimated by the urban case $\mathrm{B} \overline{\triangle P E S}$ when the maximum value $a_{\text {CUD }}$ of measurement differences is assumed to be equal to $20 \mathrm{~dB}$.

Note that in all the cases examined, $\overline{P E S_{n r}}$ is an increasing function with reference to the maximum value $a_{\text {CUD }}$. This is due to the fact the asymmetry between the modified and 
the respective original OV LV BPL topology increases as more intense measurement differences are added.

Now, as the robustness of the decisions concerning the existence of energy theft is concerned, their robustness is examined in Fig. 1(c). As indicated in Fig. 1(c), the robustness mainly depends on the maximum value $a_{\text {cuD }}$ rather than the examined OV LV BPL topology. High measurement differences create uncertainty that foments the robustness of the decisions. Very high measurement differences, which exceed $10 \mathrm{~dB}$, obviously create great uncertainty that is reflected on low values of robustness. Similarly to the $\overline{\triangle P E S}$ threshold, an arbitrary $\overline{R o b}$ threshold can also be defined by the $\overline{R o b}$ of the urban case $\mathrm{B}$ when maximum value $a_{\text {CuD }}$ of measurement differences is assumed to be equal to $20 \mathrm{~dB}$.

In numerical terms and in order to define the $\overline{\triangle P E S}$ and $\overline{R o b}$ thresholds, $\overline{\triangle P E S}$ and $\overline{R o b}$ values of the modified OV LV BPL topologies of Figs 1(a)-(c) are reported in Table 1 when maximum value $a_{\text {CuD }}$ of measurement differences is assumed to be equal to $20 \mathrm{~dB}$.

Table 1. $\overline{\triangle P E S}$ and $\overline{R o b}$ of Indicative OV LV BPL Topologies when $L_{\mathrm{bh}}=5 \mathrm{~m}$ and $D_{\mathrm{h}}=300 \mathrm{~m}$ (opencircuit hook termination) for maximum values $a_{\text {CuD }}$ equal to $20 \mathrm{~dB}$

\begin{tabular}{|c|c|c|c|c|c|}
\hline & \multicolumn{5}{|c|}{ Modified OV LV BPL Topology } \\
\hline & Urban case A & Urban case B & Suburban case & Rural case & "LOS" case \\
\hline $\begin{array}{c}\overline{\overline{\triangle P E S}} \\
(\%)\end{array}$ & 15.47 & 10.80 & 24.73 & 37.90 & 155.55 \\
\hline $\begin{array}{l}\overline{R o b} \\
(\%)\end{array}$ & 24.01 & 23.42 & 28.46 & 29.05 & 41.06 \\
\hline
\end{tabular}

From Table 1, it is evident that HS-DET method can safely detect the energy theft even if the worst scenarios concerning the examined OV LV BPL topology and measurement differences are assumed. Also, a strict version of $\overline{\triangle P E S}$ and $\overline{R o b}$ thresholds can be assumed to be equal to $10 \%$ and $20 \%$, respectively. Finally, as it has been already recognized by Figs. 1(a)-(c), urban case B and "LOS" case define the worst and the best case regarding the energy theft detection in OV LV BPL networks when high measurement differences are assumed.

In order to understand the potential reader the strict version of $\overline{\triangle P E S}$ and $\overline{R o b}$ thresholds as well as the safety that is provided, the impact of measurement differences during the computation of $\overline{\triangle P E S}, \overline{P E S_{\mathrm{nr}}}$ and $\overline{R o b}$ of the original indicative OV LV BPL topologies is given in the Appendix. Instead of a modified OV LV BPL topology with a hook, it is assumed that the modified OV LV BPL topology is the indicative OV LV BPL topology. It is proven there that the assumed $\overline{\triangle P E S}$ and $\overline{R o b}$ thresholds are high enough in order not to active a fault energy theft alarm because of high measurement differences.

The high measurement differences, which are assumed in this paper, can be either intentionally or unintentionally occur. However, the HS-DET method is robust enough and can act as countermeasure technique in order to mitigate these measurement differences. If all the other problematic cases, which concern the operation of the OV LV power grid and described in [2]-[8], are excluded, HS-DET method can safely detect hook style energy thefts even though measurement differences up to $20 \mathrm{~dB}$ are assumed 
due to the countermeasure character against measurement differences of HS-DET method (see the Appendix).

However, intentional measurement differences can be combined with other techniques in order to mask a potential hook style energy theft. In the following subsections, the installation of very long hooks (see Sec.3.2) and the use of "smart" hooks (see Sec.3.3) that are matched to the characteristic impedances of the lines are examined in order to jam HS-DET method when intentional measurement differences are produced.

\subsection{Very Long Hooks and HS-DET Method Jamming}

The influence of measurement differences of maximum value $a_{\text {CuD }}$ above $5 \mathrm{~dB}$ has been presented in Sec.3.1. The installation of very long hooks is considered as one of the potential techniques against the energy theft detection. In this subsection, the detection performance of HS-DET method is investigated when the technique of very long hooks is adopted and measurement differences occur.

To examine, the detection performance of HS-DET method, in Fig. 2(a), $\overline{\triangle P E S}$ is plotted versus the hook distance from the transmitting end and the hook length when the OV LV BPL topology of urban case A is assumed, very long hooks are considered (i.e., $50 \mathrm{~m}, 100 \mathrm{~m}, 200 \mathrm{~m}, 500 \mathrm{~m}, 1000 \mathrm{~m}, 2000 \mathrm{~m}$ and $5000 \mathrm{~m})$ and maximum value $a_{\text {CuD }}$ of $1 \mathrm{~dB}$ is applied. In Figs. 2(b)-(d), same contour plots with Fig. 2(a) are given but for maximum value $a_{\text {cuD }}$ of $5 \mathrm{~dB}, 10 \mathrm{~dB}$ and $20 \mathrm{~dB}$, respectively. In Figs. 3, 4, 5 and 6, same plots with Fig. 2 are given but for the case of the urban case B, suburban case, rural case and "LOS" case, respectively. In accordance with [1], the hook distance from the transmitting end span is assumed to be equal to $50 \mathrm{~m}$ while the range of the hook distance from the transmitting end is from $1 \mathrm{~m}$ to $951 \mathrm{~m}$ for all the examined contour plots of this paper. 


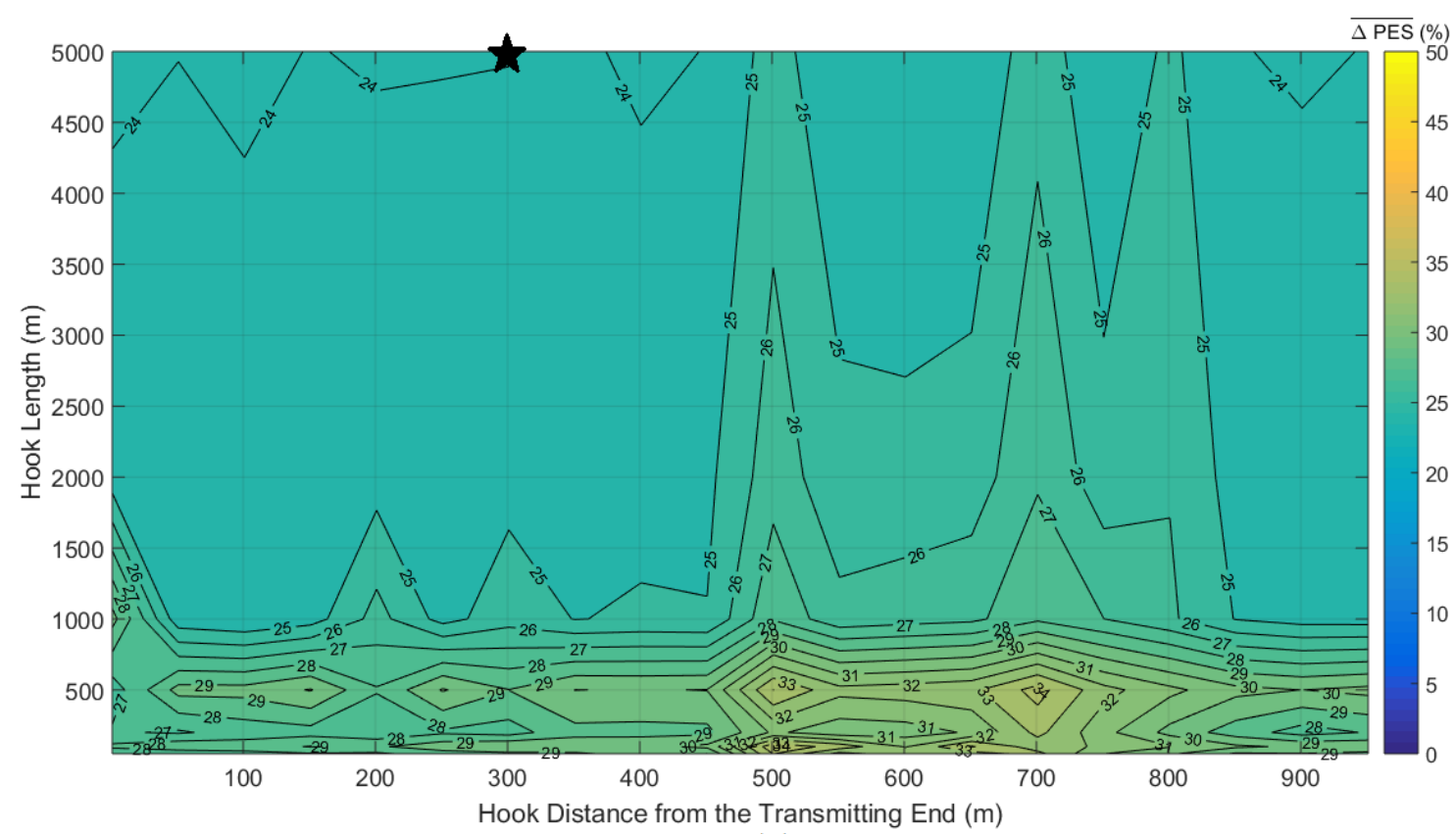

(a)

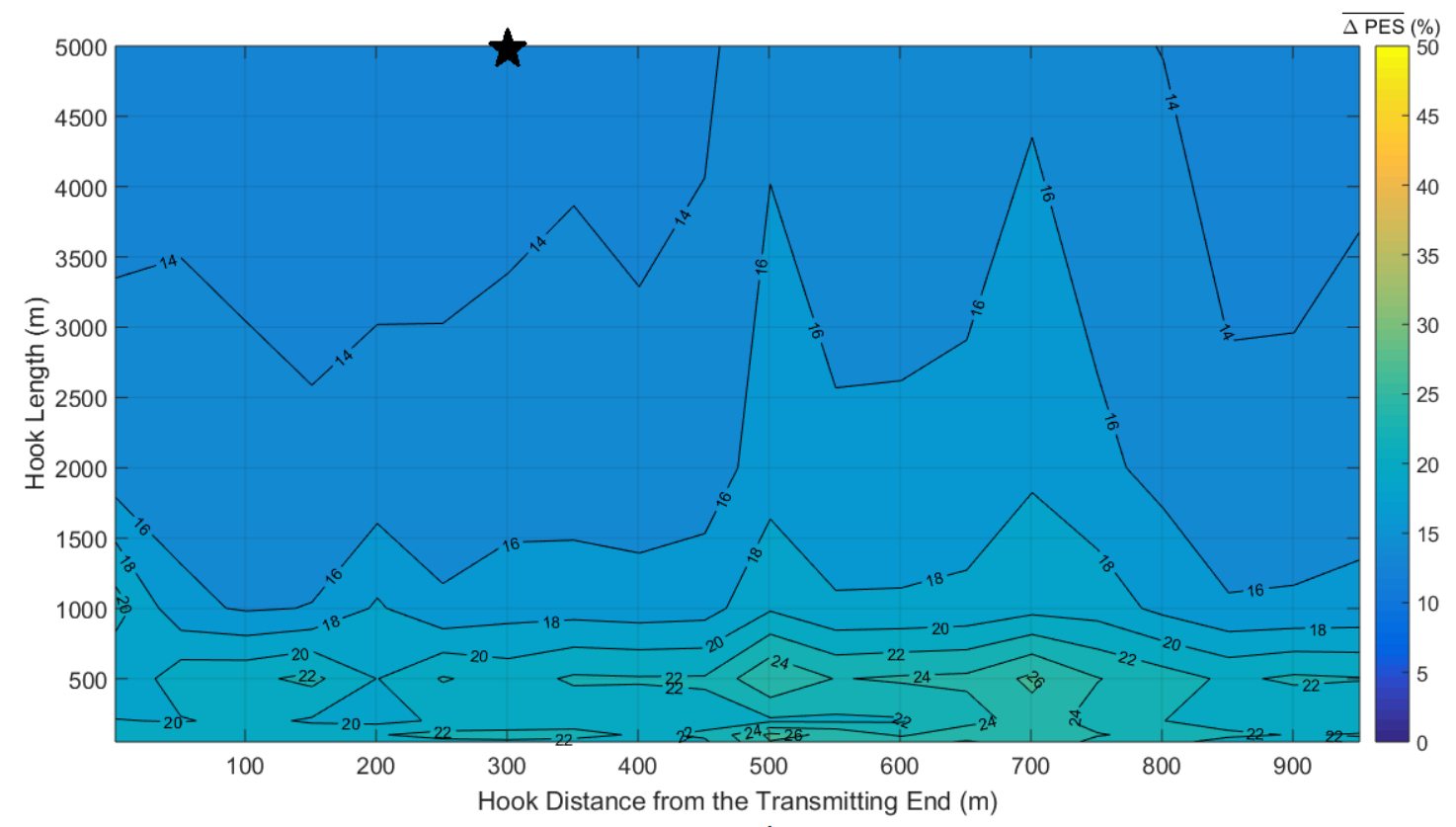

(b) 


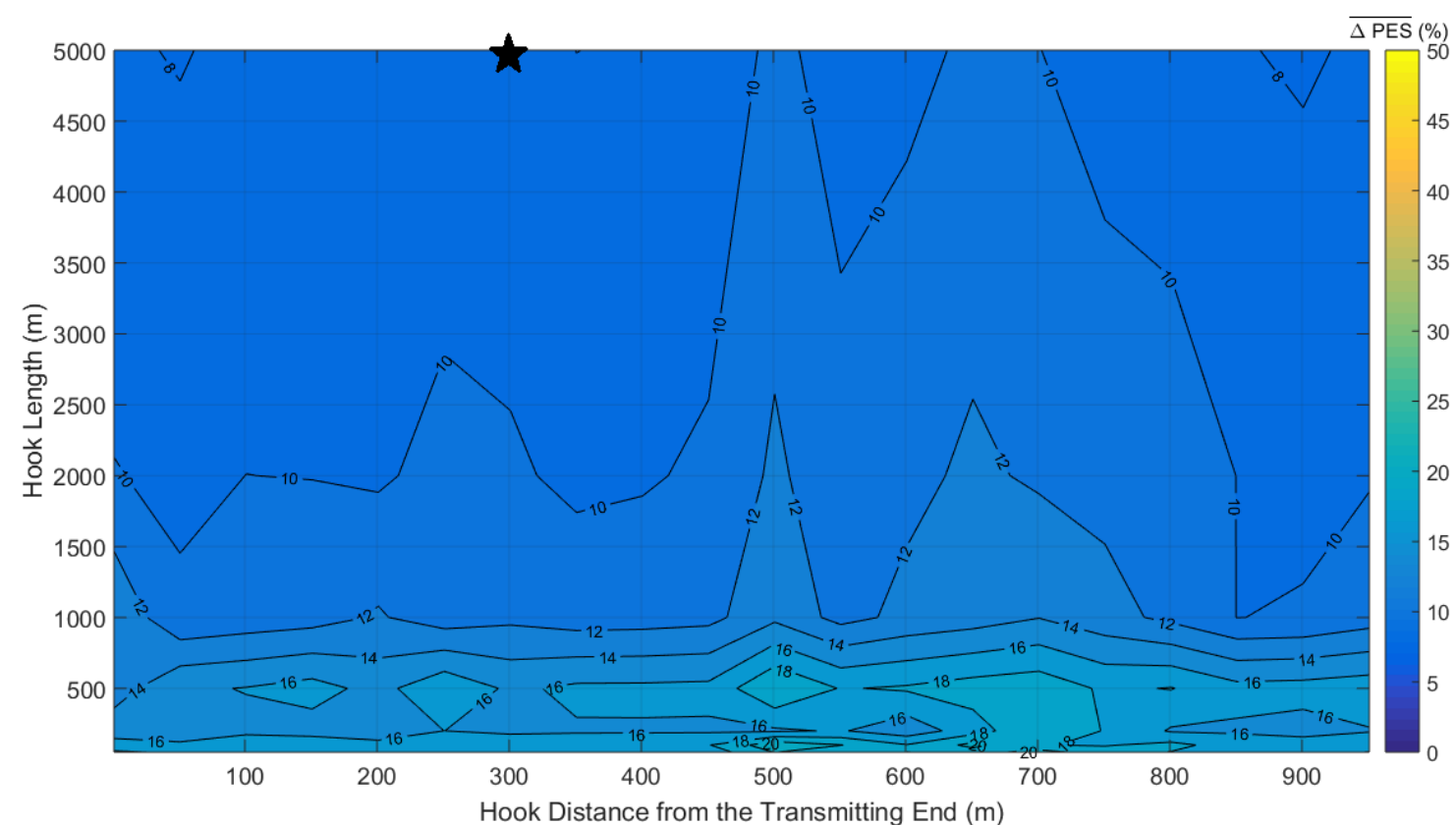

(c)

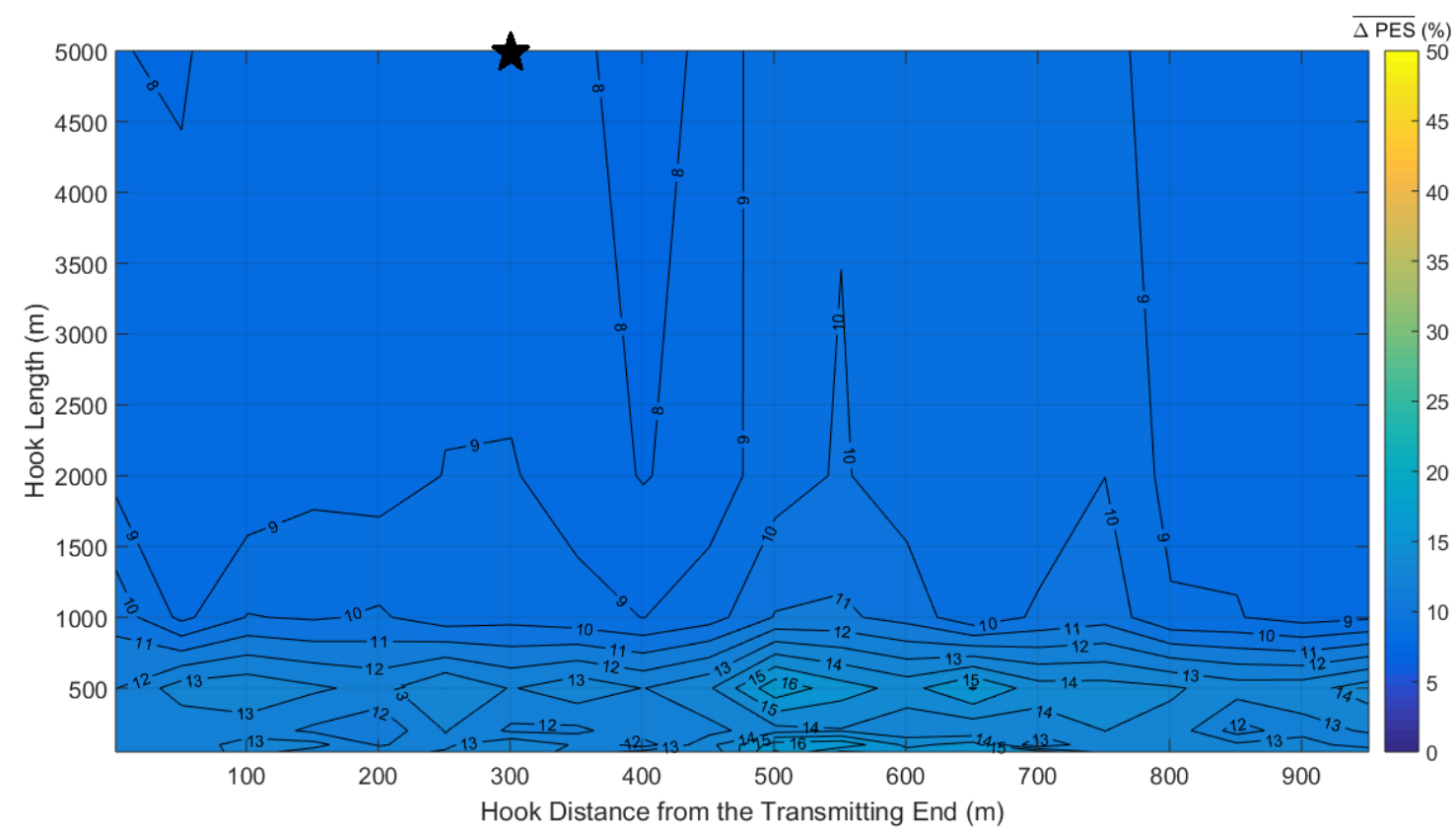

(d)

Fig. 2. $\overline{\triangle P E S}$ of the urban case A of the indicative OV LV BPL topologies in the 3-88 MHz frequency band for various hook distances from the transmitting end and very long hook lengths when high maximum values $a_{\text {CUD }}$ are assumed. (a) $a_{\text {CUD }}=1 \mathrm{~dB}$. (b) $a_{\text {CUD }}=5 \mathrm{~dB}$. (c) $a_{\text {CUD }}=10 \mathrm{~dB}$. (d) $a_{\text {CUD }}=20 \mathrm{~dB}$. 


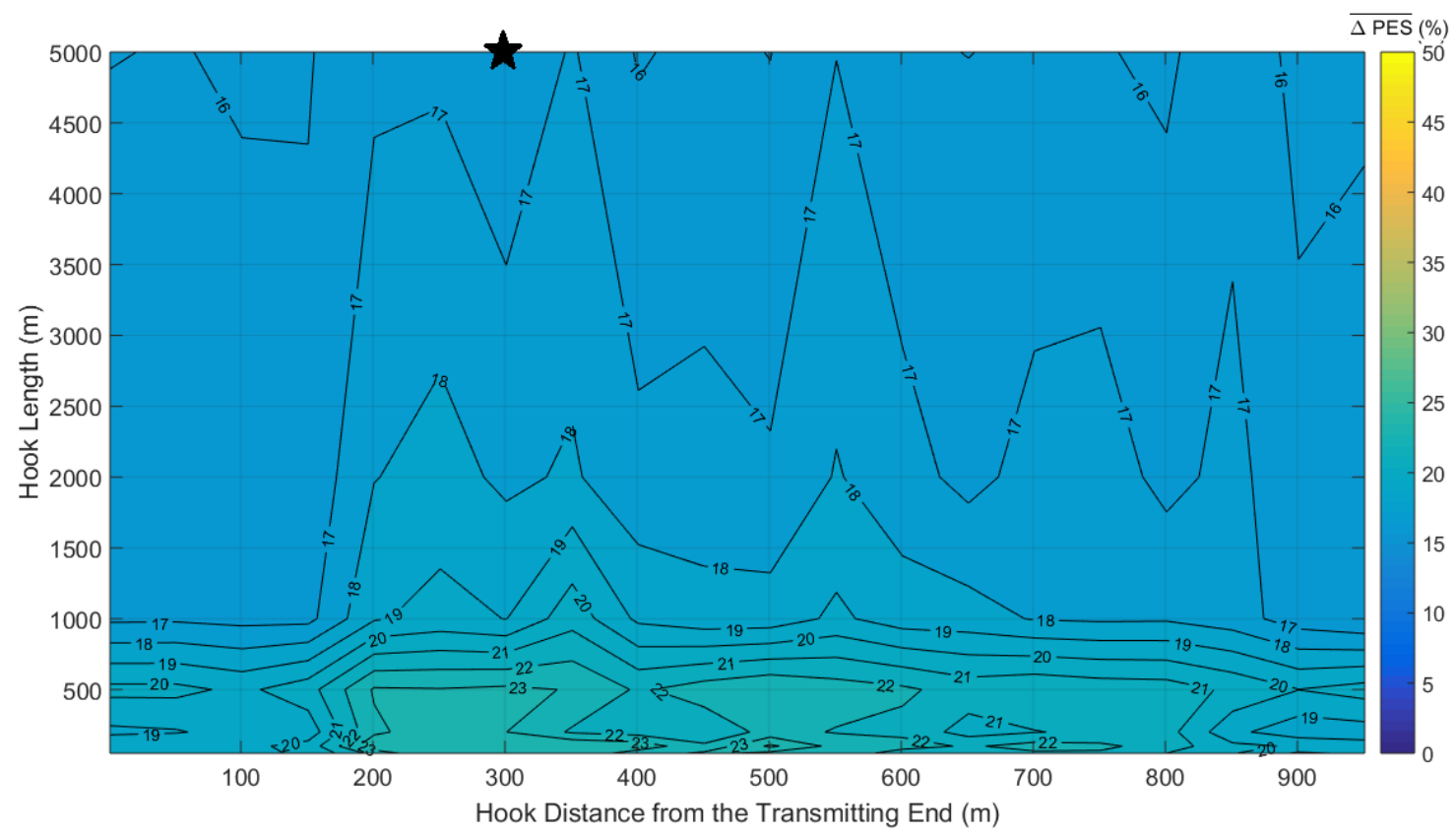

(a)

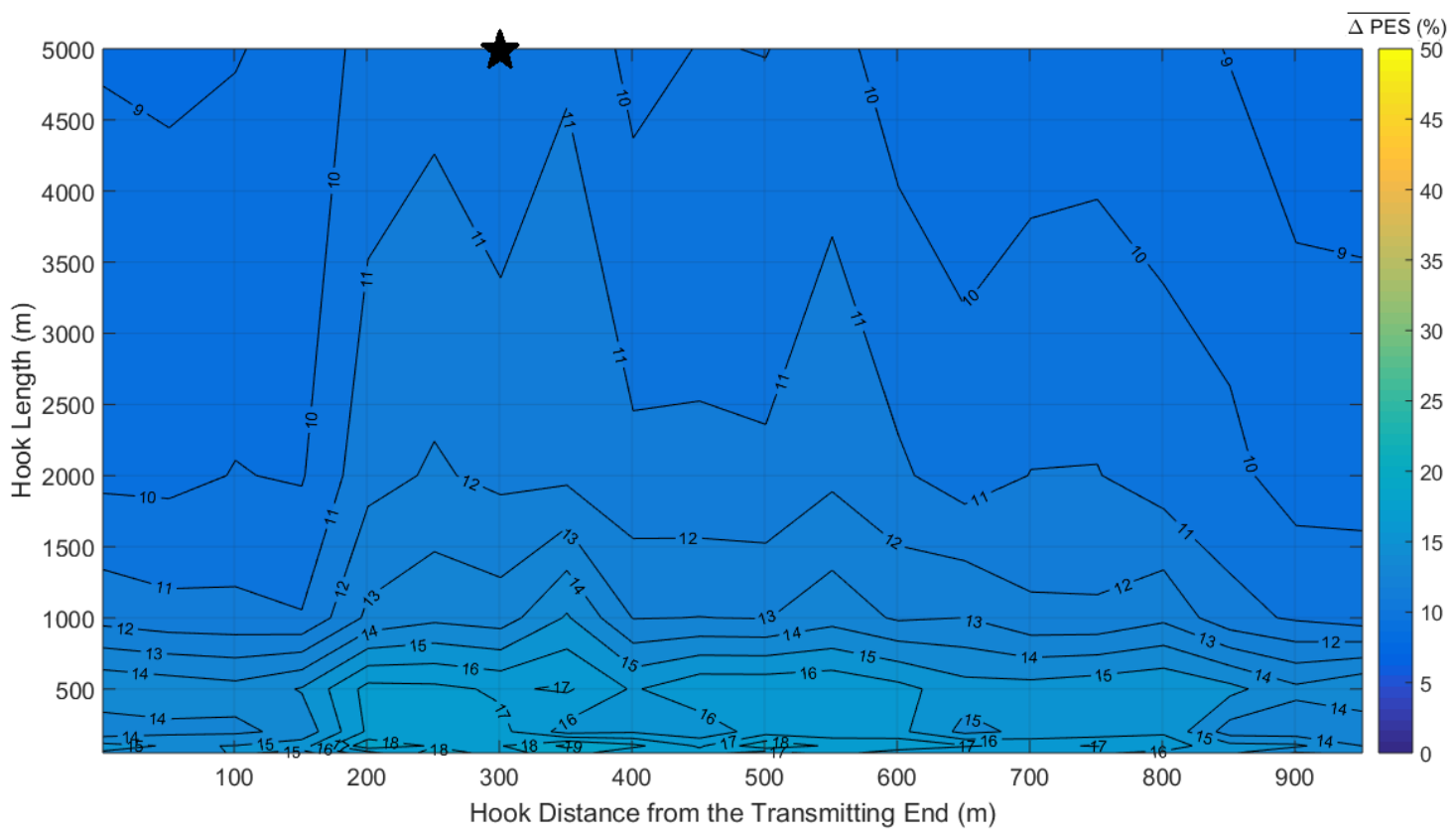

(b) 


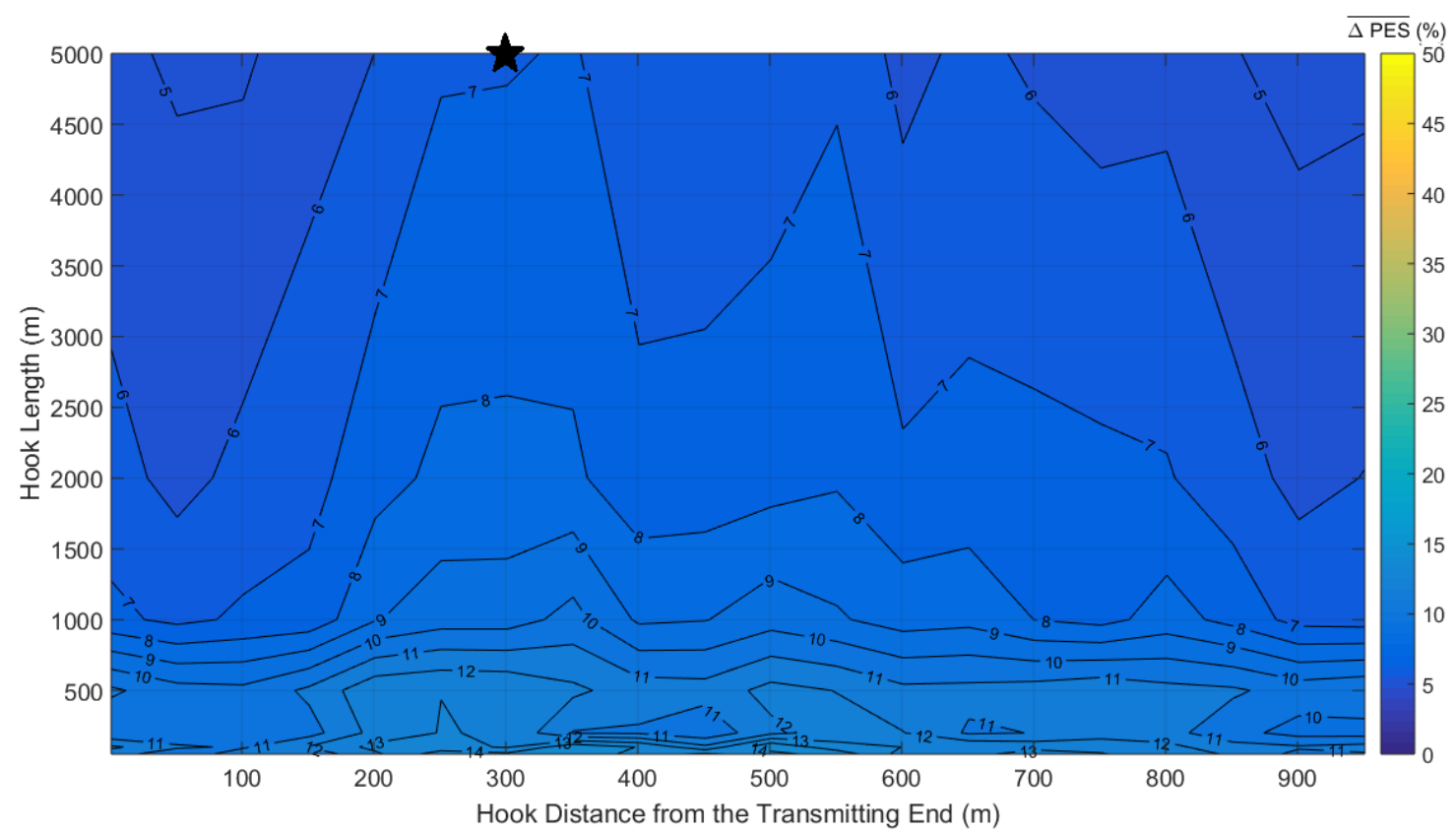

(c)

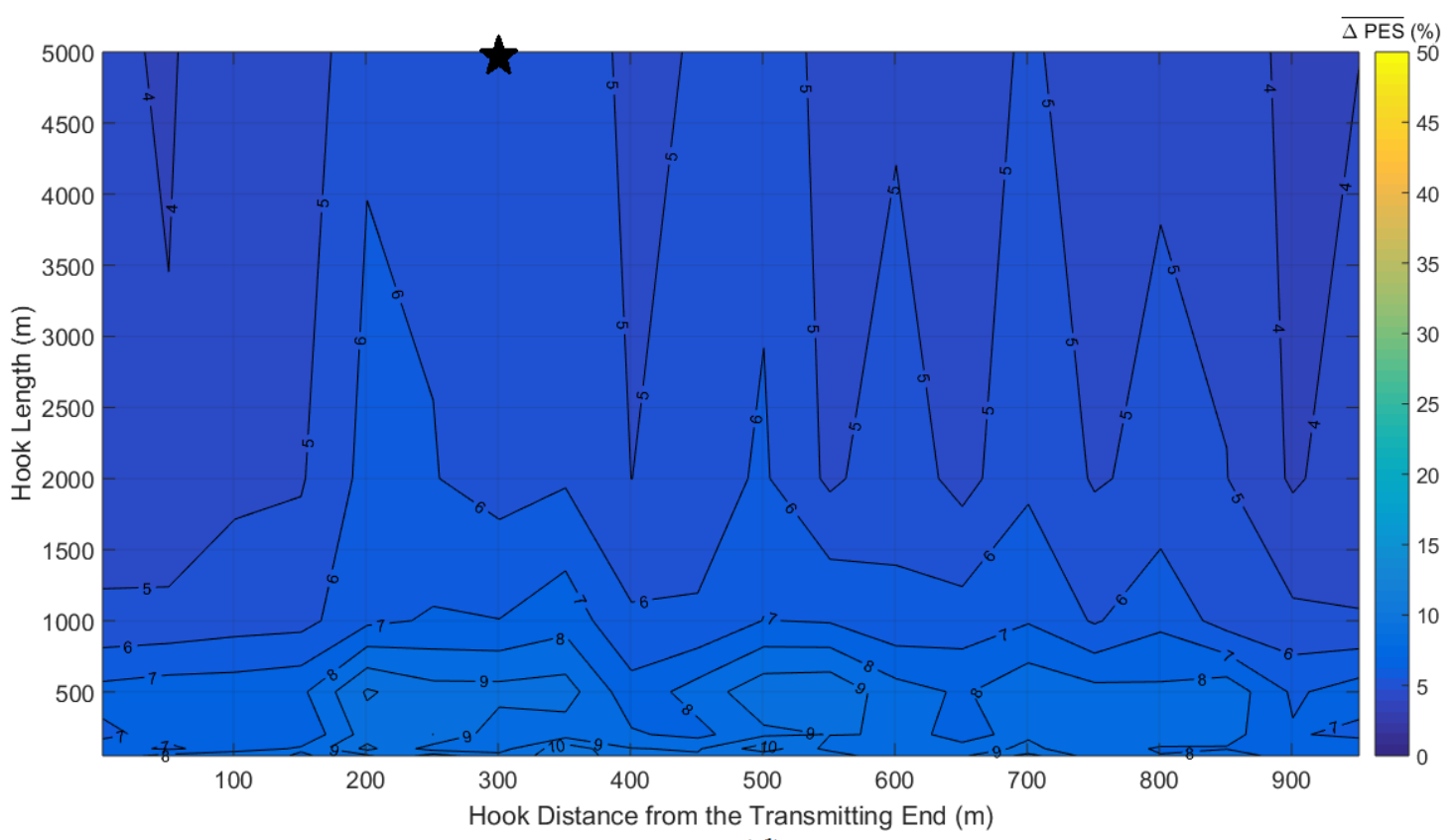

(d)

Fig. 3. Same curves with Fig. 2 but for the urban case B. 


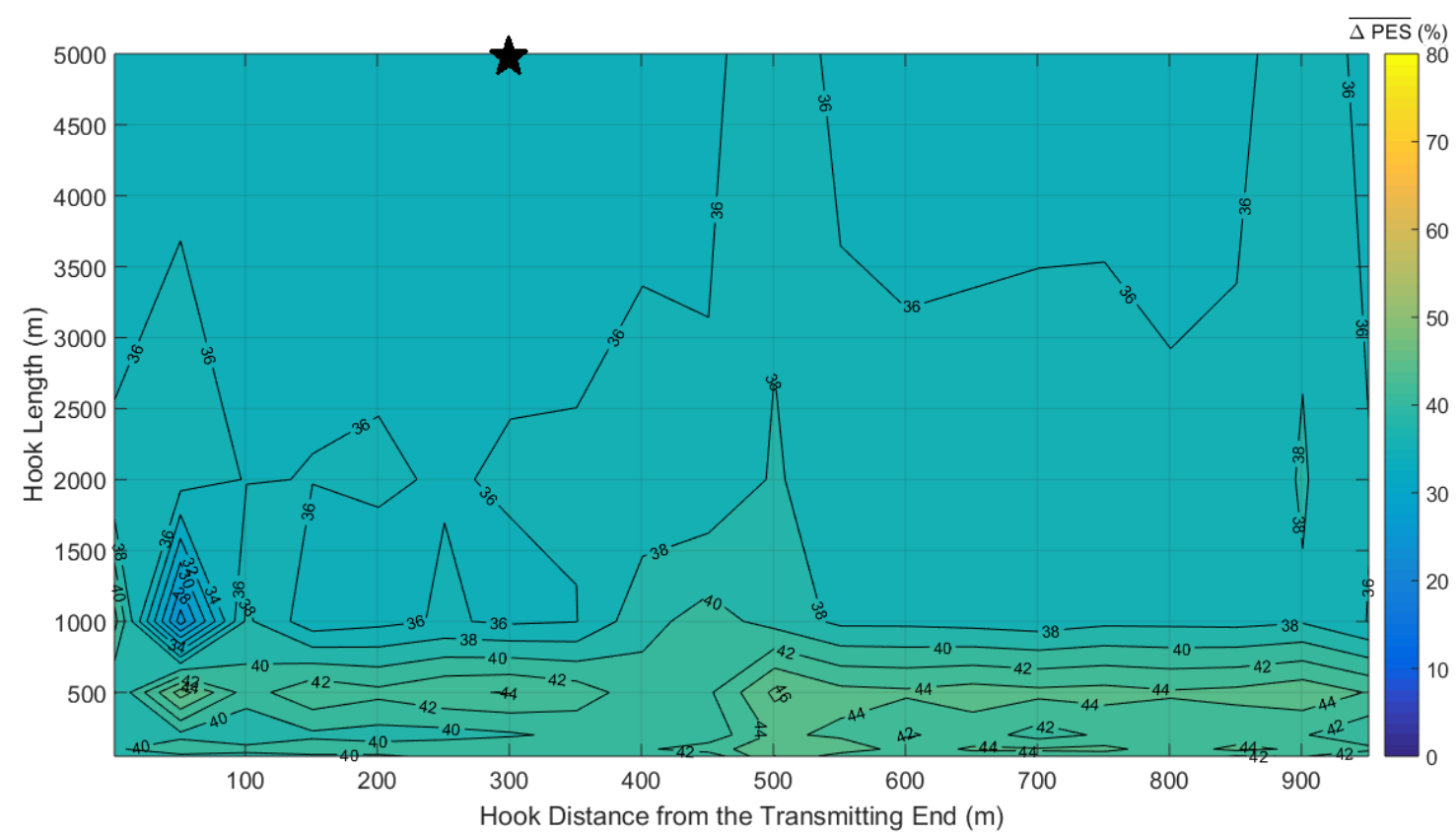

(a)

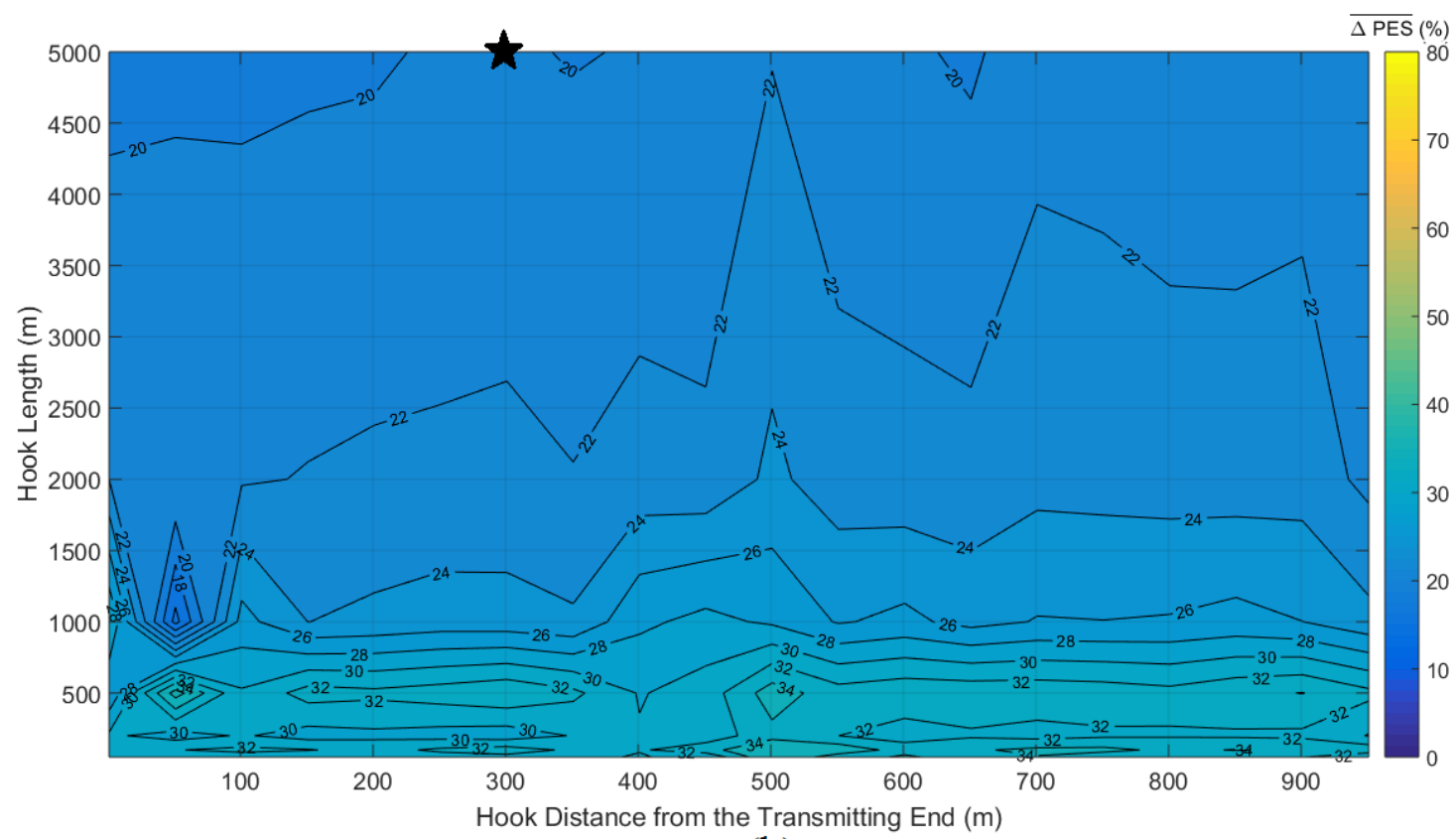

(b) 

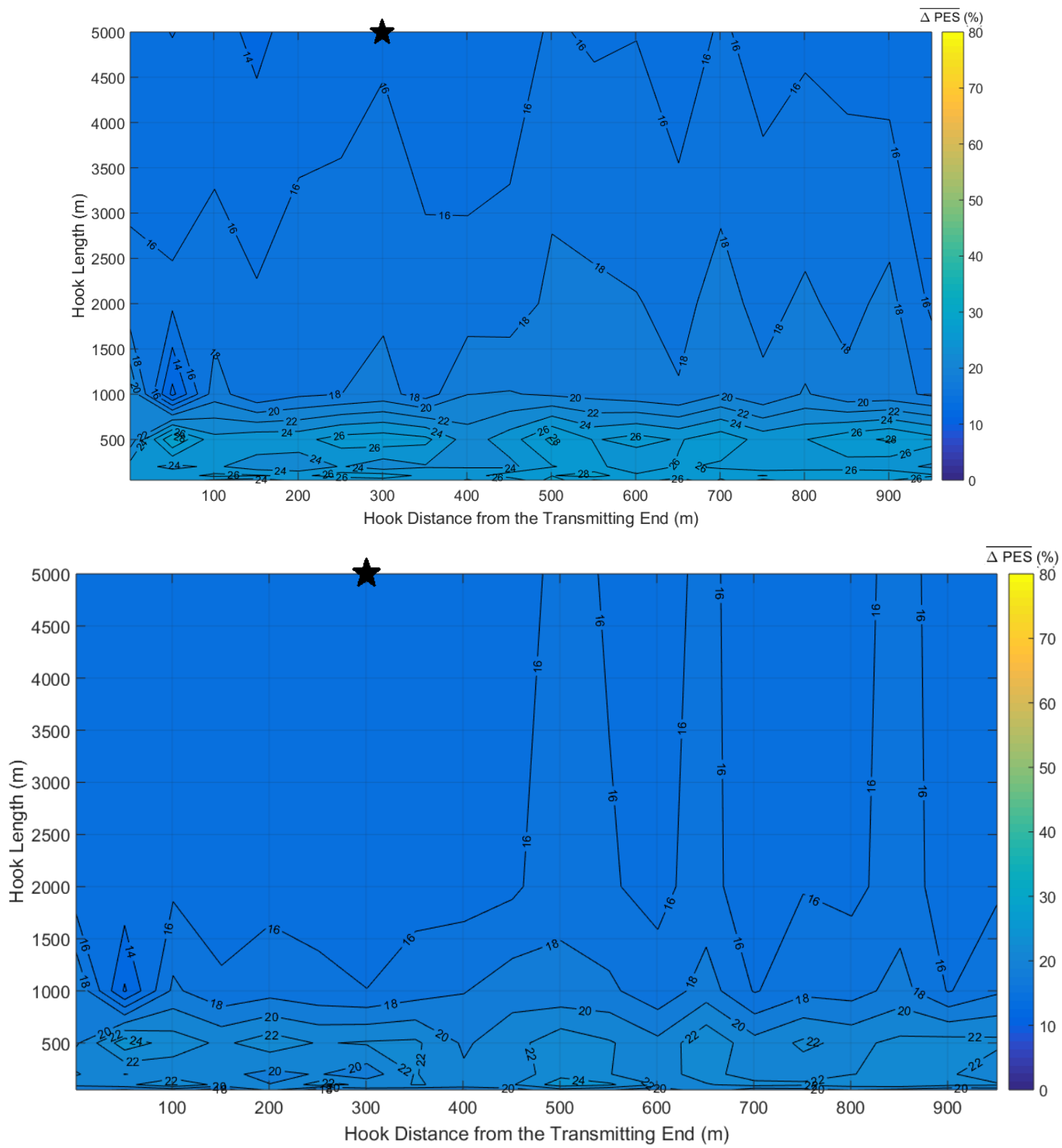

(d)

Fig. 4. Same curves with Fig. 2 but for the suburban case. 


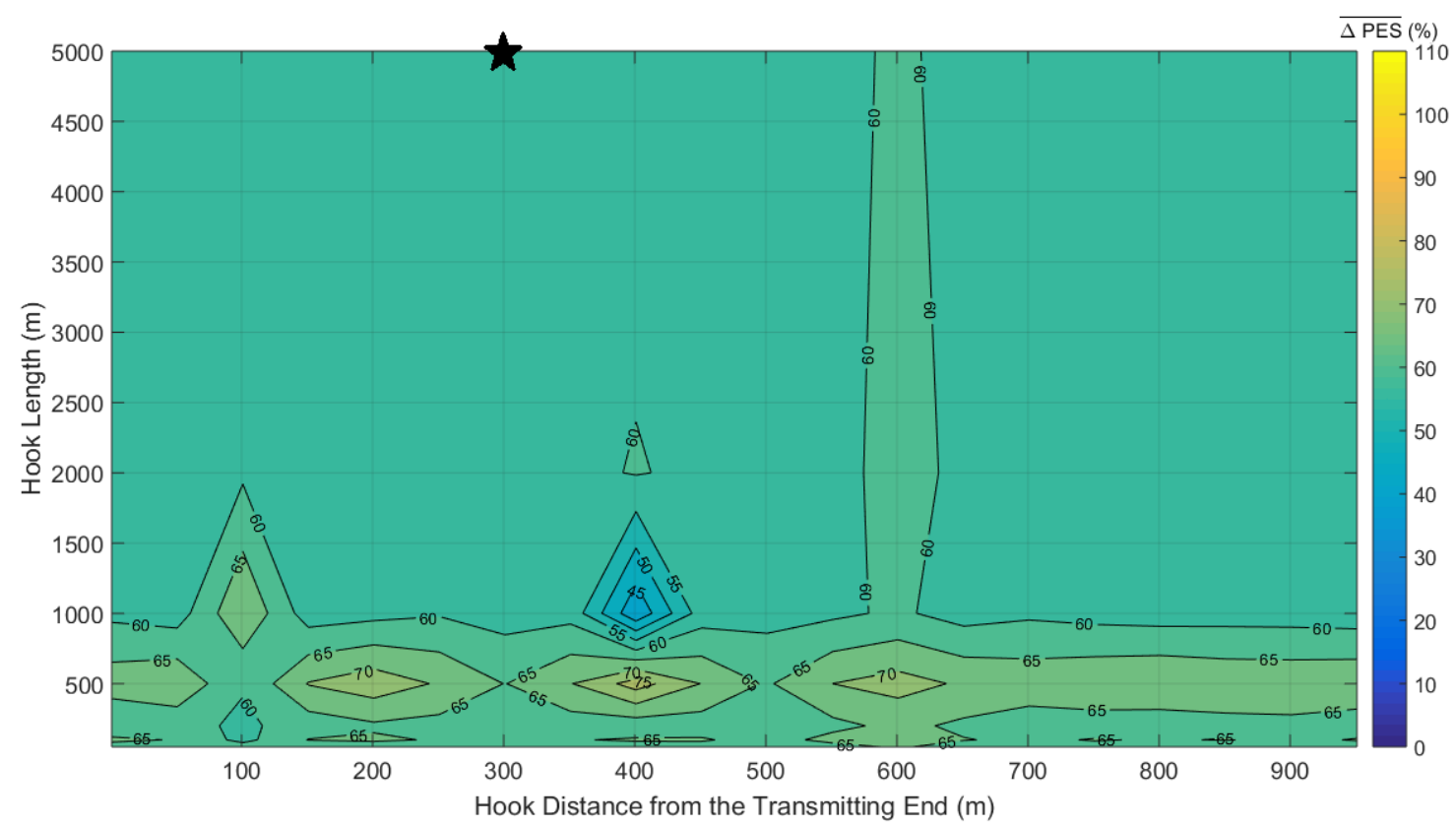

(a)

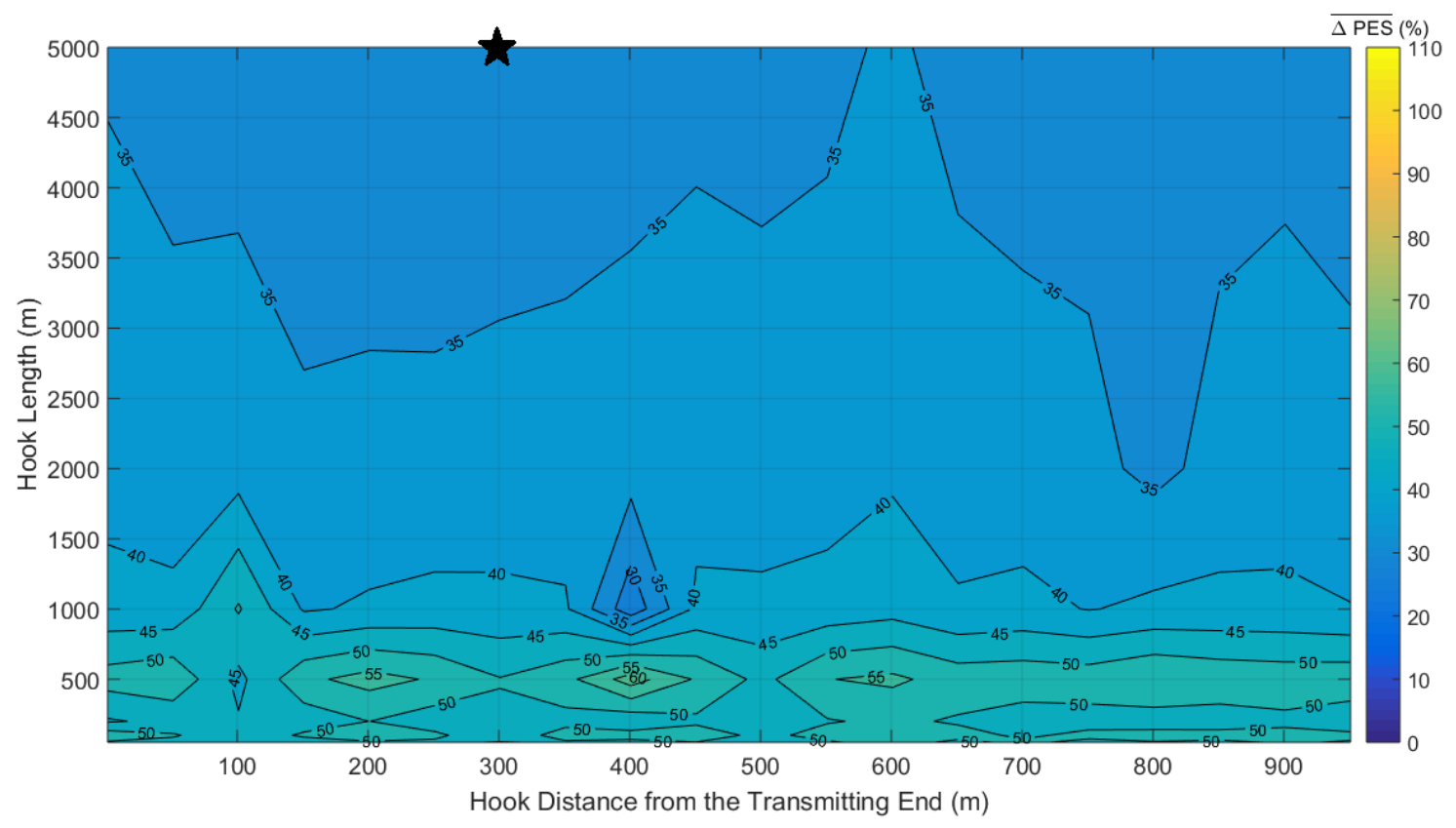

(b) 


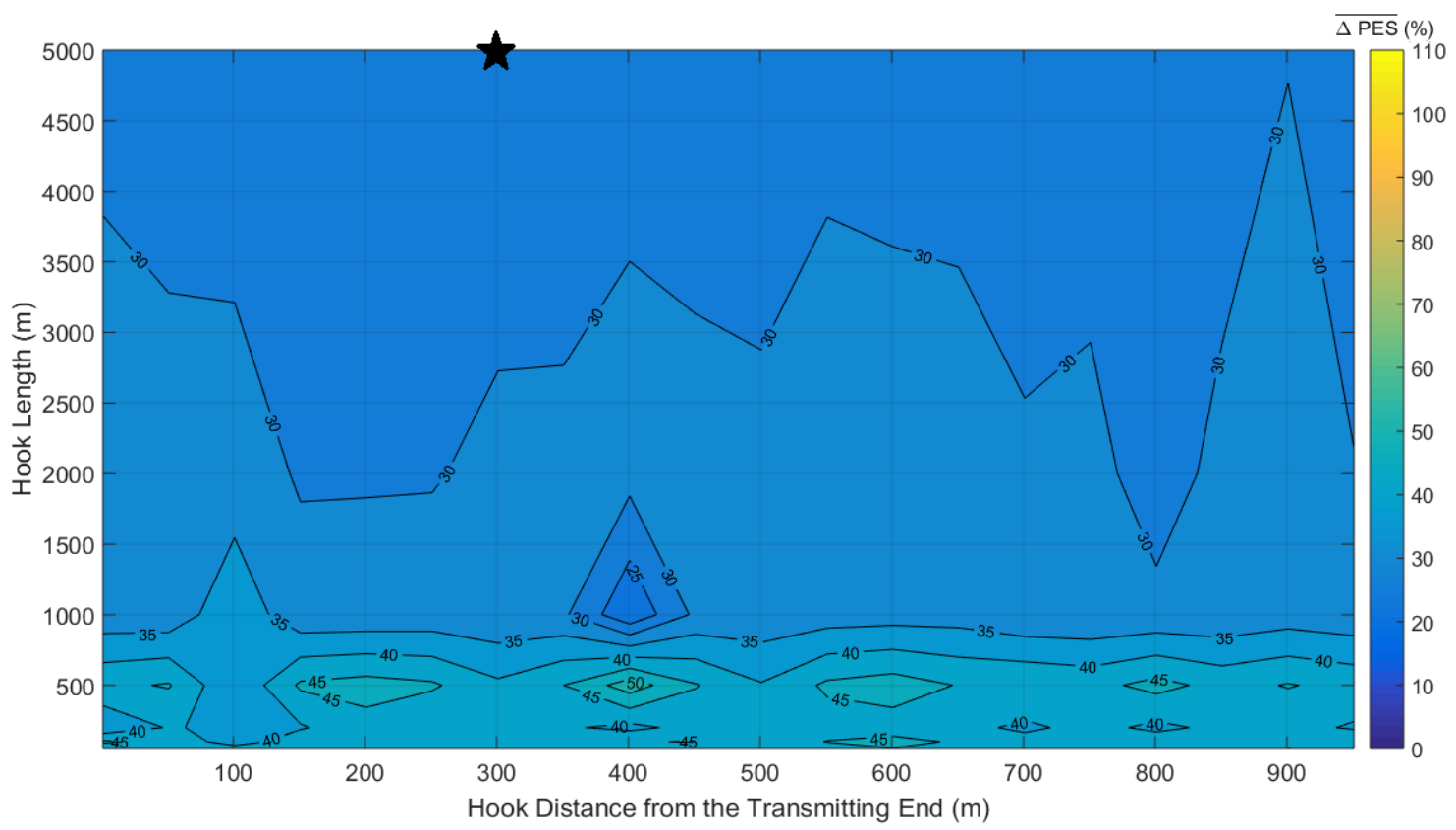

(c)

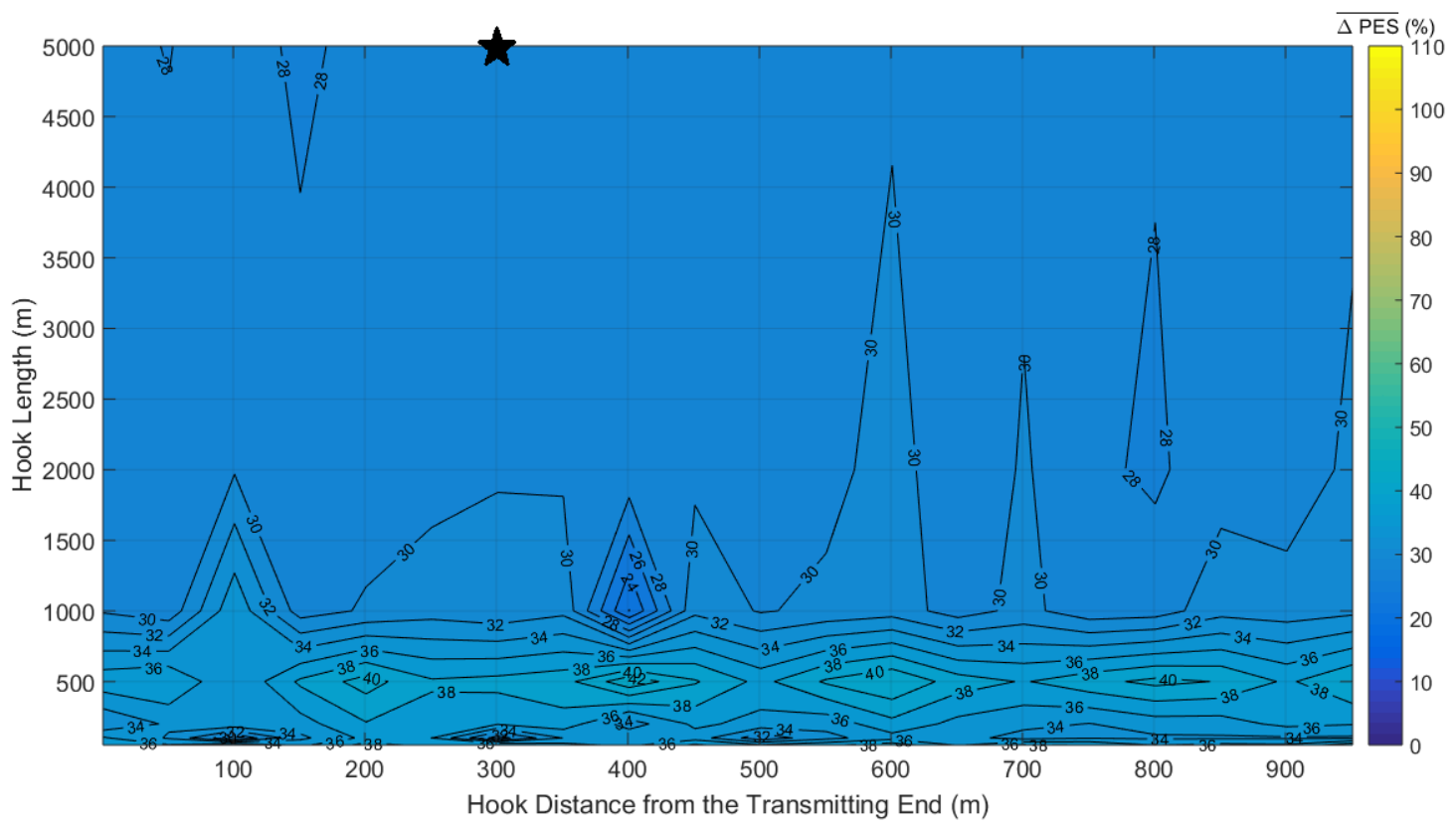

(d)

Fig. 5. Same curves with Fig. 2 but for the rural case. 


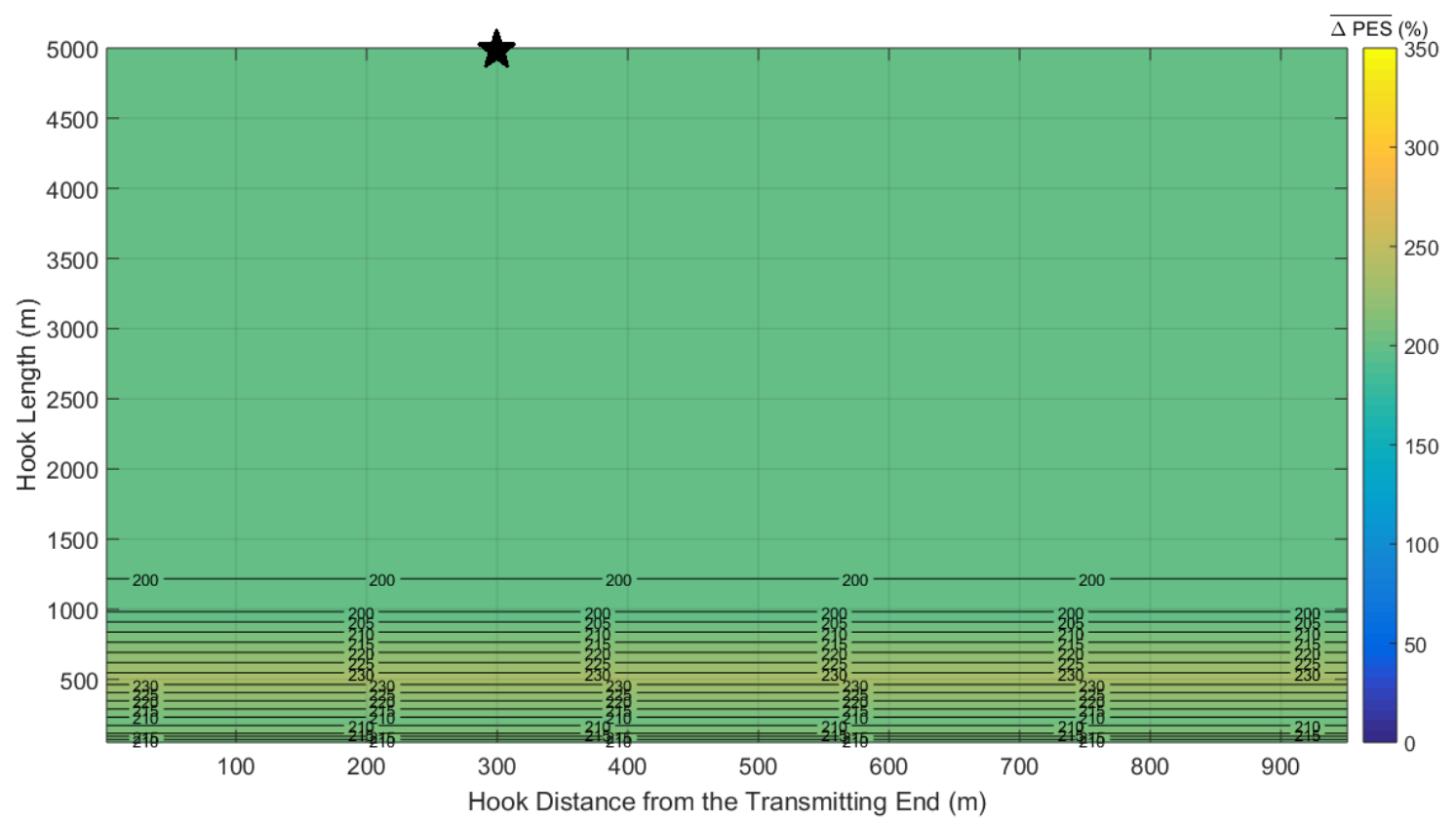

(a)

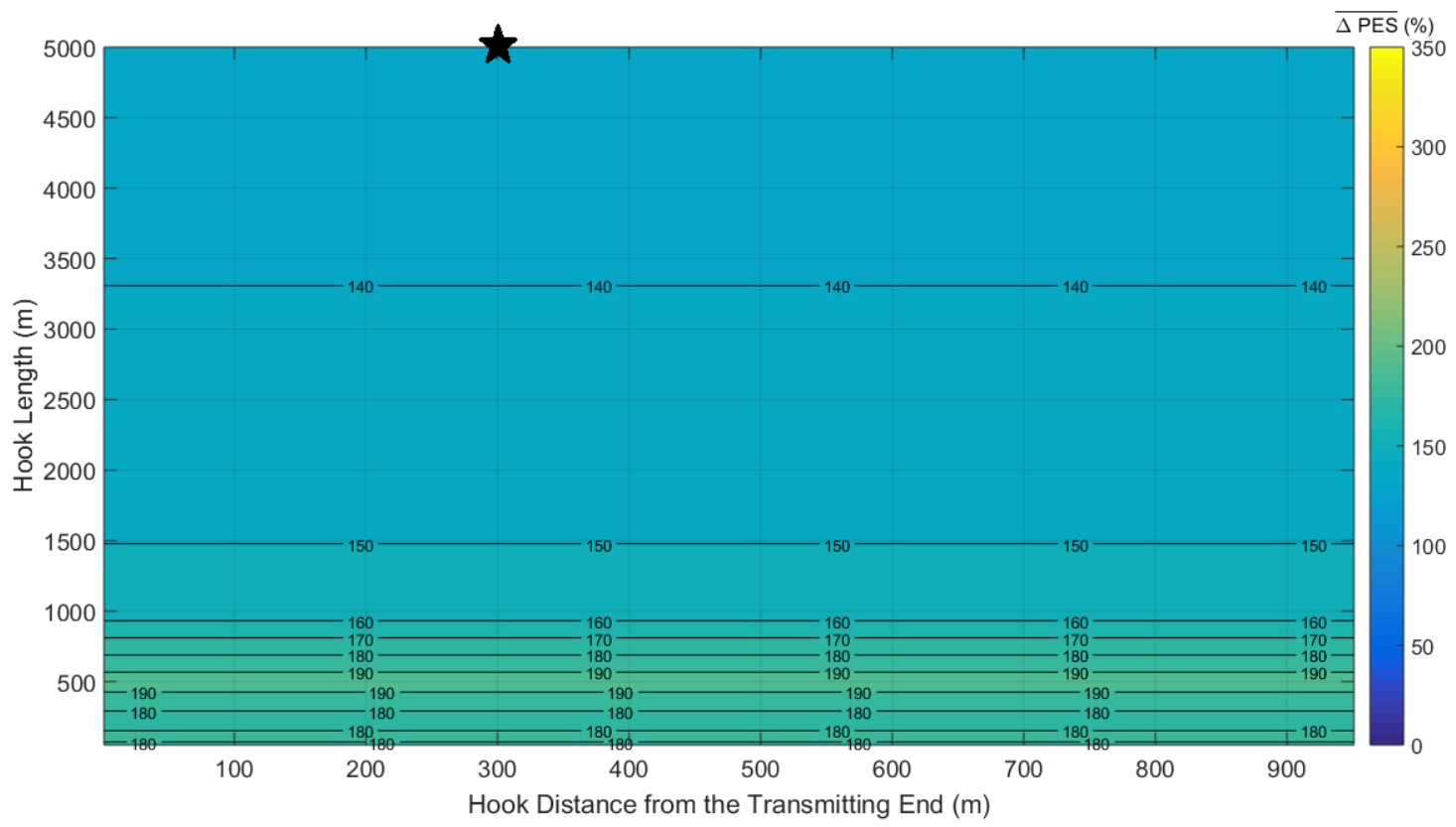

(b) 


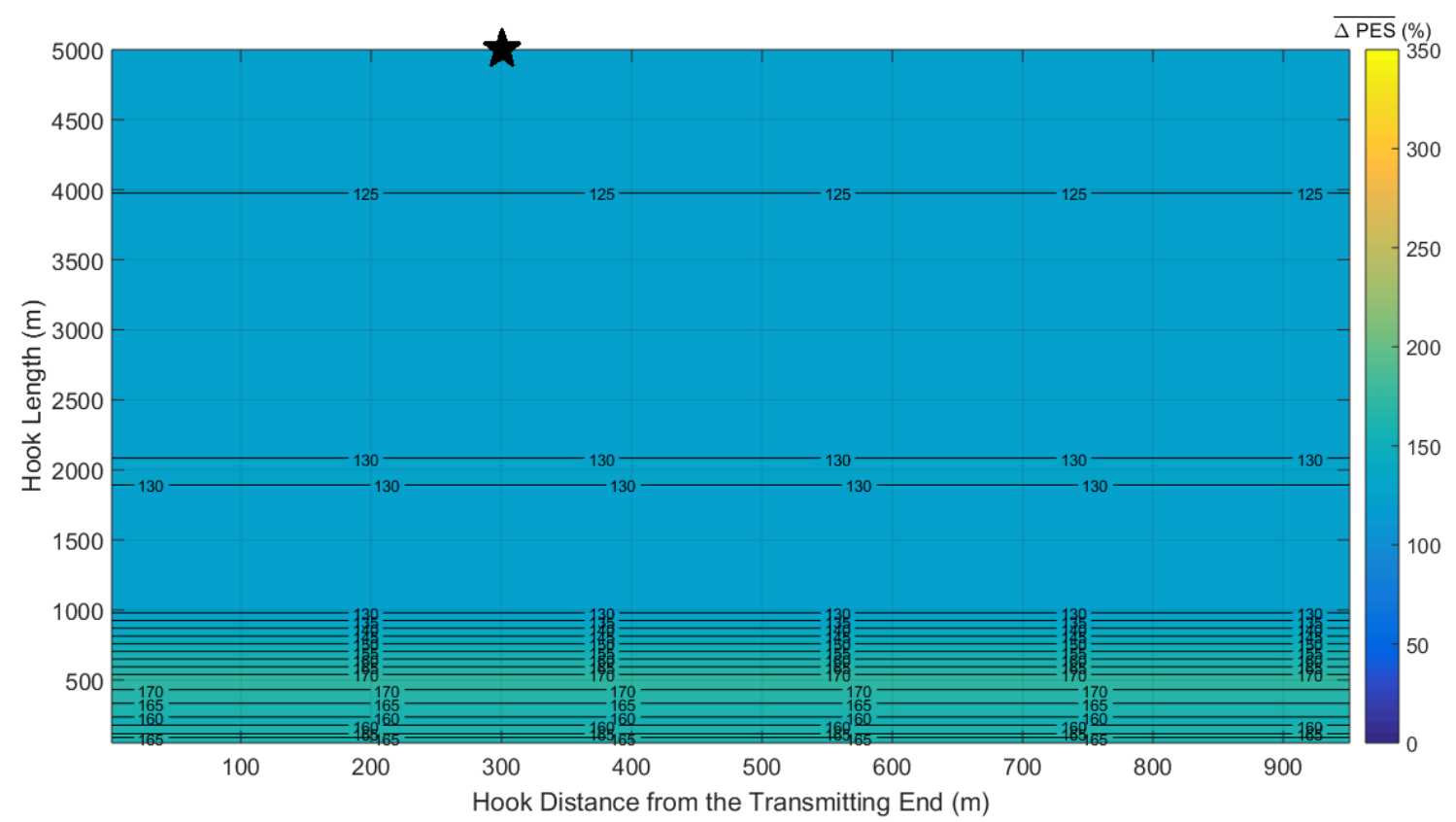

(c)

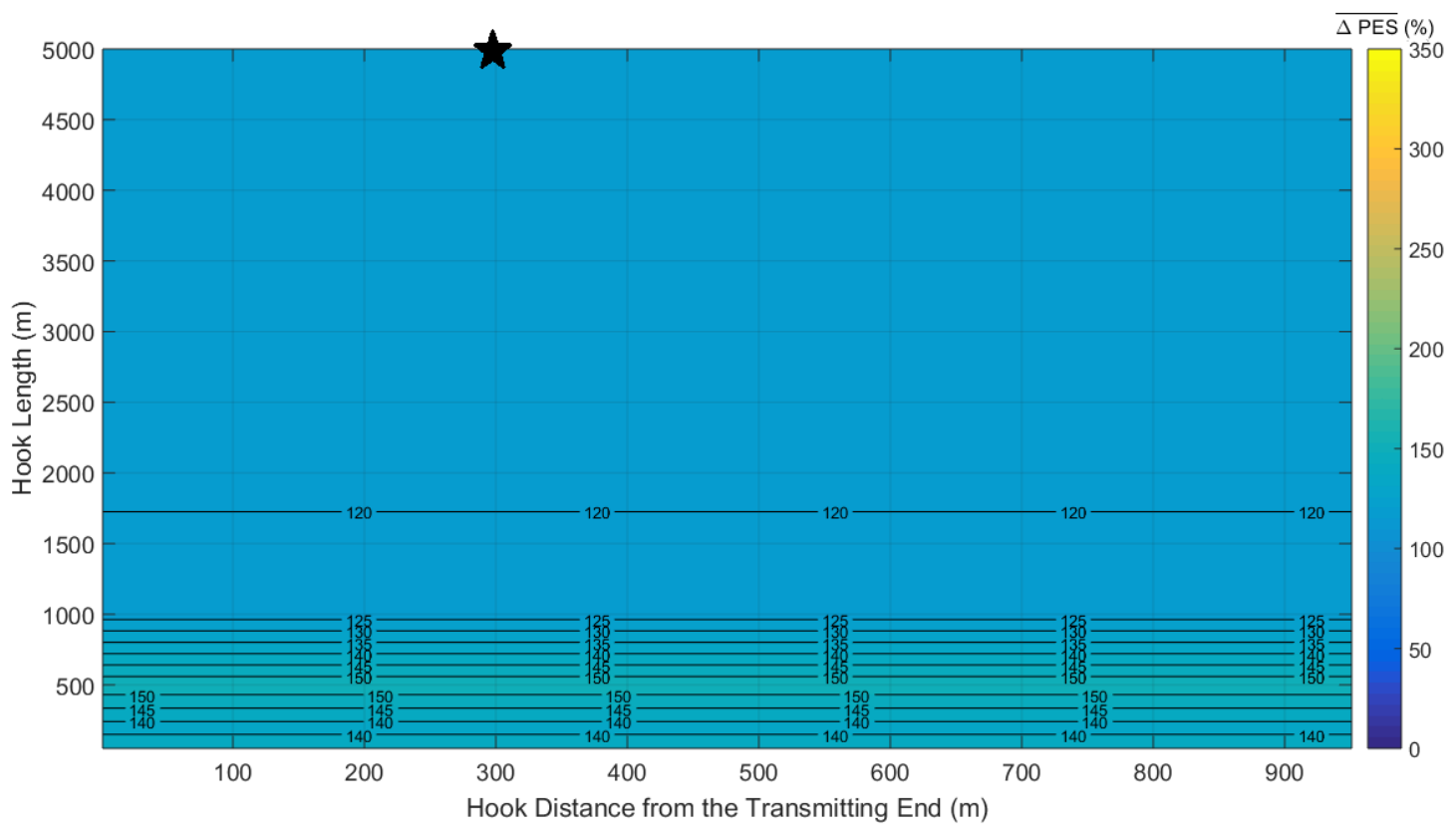

(d)

Fig. 6. Same curves with Fig. 2 but for the "LOS" case.

From Figs. 2-6, several interesting conclusions concerning the technique of the very long hooks that aims at jamming HS-DET method can be reported, namely:

- As in [1] and already reported in Table 1, high measurement differences significantly influence the values of $\overline{\triangle P E S}$ regardless of the examined OV LV BPL topology, the hook distance from the transmitting end and the hook length. Contour plots of colder colors occur as the maximum value of measurement differences increases implying that the combination of any energy theft technique 
with high measurement differences renders the detection of the energy theft more difficult.

- When very long hooks are applied, $\overline{\triangle P E S}$ receives significantly lower values in comparison with $\overline{\triangle P E S}$ values of short hooks. Since long hooks are treated by the hybrid model as branches, long branches create shallow and rare notches in coupling transfer functions. In accordance with TL theory, while the hooks are characterized by very high length, their input impedance tends to be matched. Therefore, these pseudo-matched terminations can indeed challenge HS-DET method through their lower $\overline{\triangle P E S}$ values of their modified OV LV BPL topologies in comparison with the respective topologies of short length hook and open-circuit termination. Hence, hook lengths that tend to infinity create little $\overline{\triangle P E S}$ difference in comparison with the hook lengths of $5000 \mathrm{~m}$ since they anyway resemble to a matched termination.

- When very long hooks are installed, the main difficulty of HS-DET method concerning the detection of the energy theft is focused on the multi-branched OV LV BPL topologies. Actually, when very long hooks are installed in urban OV LV BPL topologies (e.g., urban case A and B) and are combined with high measurement differences, $\overline{\triangle P E S}$ values of these modified urban cases can become lower than the strict $\overline{\triangle P E S}$ threshold of $10 \%$ of HS-DET method in some cases. For example, $\overline{\triangle P E S}$ islands of $9 \%, 5 \%$ and $4 \%$ appear in $\overline{\triangle P E S}$ contour plots of the modified urban case $\mathrm{B}$ when maximum value $a_{\text {CuD }}$ is assumed to be equal to $5 \mathrm{~dB}, 10 \mathrm{~dB}$ and $20 \mathrm{~dB}$, respectively. Anyway, the energy theft can be detected by HS-DET method through its loose $\overline{\triangle P E S}$ threshold of $0 \%$.

- The most emphatic performance difference of HS-DET method when very long branches are deployed can be revealed by comparing Fig. 8(a) of [1] and Fig. 6(a) of this paper. $\overline{\triangle P E S}$ differences up to $100 \%$ can be spotted when a very long hook is hung at the MTL configuration of the "LOS" OV LV BPL topology.

- Although the energy theft through very long hooks can make its detection less easy by HS-DET method, its practicality is under question. Issues concerning the power delivery quality, the power attenuation as well as the size of the energy theft installation make the adoption of this technique practically less possible.

On the basis of the matched terminations that can jam HS-DET method in the extent discussed before, an interesting trade-off between the hook length and the hook smartness can be defined. Say, instead of using very long hooks that resemble matched terminations but they are easily detectable, smart hooks that can be configured to be matched to the characteristic impedance of the examined OV LV BPL topology can be used.

\subsection{Smart Hooks and HS-DET Method Jamming}

Although the installation of additional equipment on the power grid is easily detectable by the authorized maintenance personnel and the energy theft cannot live life long, smart hooks can be camouflaged due to their smaller size in comparison with the very long hooks technique thus saving sufficient time until their detection. Actually, smart hooks can be hooks of relatively short length that are matched to the characteristic impedance in the 3-88 MHz frequency band of BPL operation. 
As been observed in Figs. 2-6, HS-DET method can detect any hook style energy theft that is based on the concept of matched terminations. Regardless of the technique that is used to accomplish matched terminations (i.e., either via very long hooks or via smart hooks), HS-DET method can detect the energy theft: (i) in the majority of the cases through its strict $\overline{\triangle P E S}$ threshold; and (ii) in the cases of the aggravated urban OV LV BPL topologies that suffer from intentional / unintentional measurement differences through the loose $\overline{\triangle P E S}$ threshold.

It is expected that the smart hooks will present the same $\overline{\triangle P E S}$ behavior with the cases examined in Figs. 2-6 where hook lengths of $5000 \mathrm{~m}$ occur for given OV LV BPL topology. Indeed, similarly to Sec.3.1, let assume that a smart hook (i.e., matched termination) of length $L_{\mathrm{bh}}$ is inserted at distance $D_{\mathrm{h}}$ from the transmitting end. In Fig. $7(\mathrm{a}), \overline{\triangle P E S}$ is plotted with respect to the maximum value $a_{\mathrm{CUD}}$ when $L_{\mathrm{bh}}=5 \mathrm{~m}$ and $D_{\mathrm{h}}=300 \mathrm{~m}$ are assumed for the five indicative original OV LV BPL topologies. In Figs. 7(b) and 7(c), same curves with Fig. 7(a) are given but for $\overline{P E S_{\mathrm{nr}}}$ and $\overline{R o b}$, respectively. 


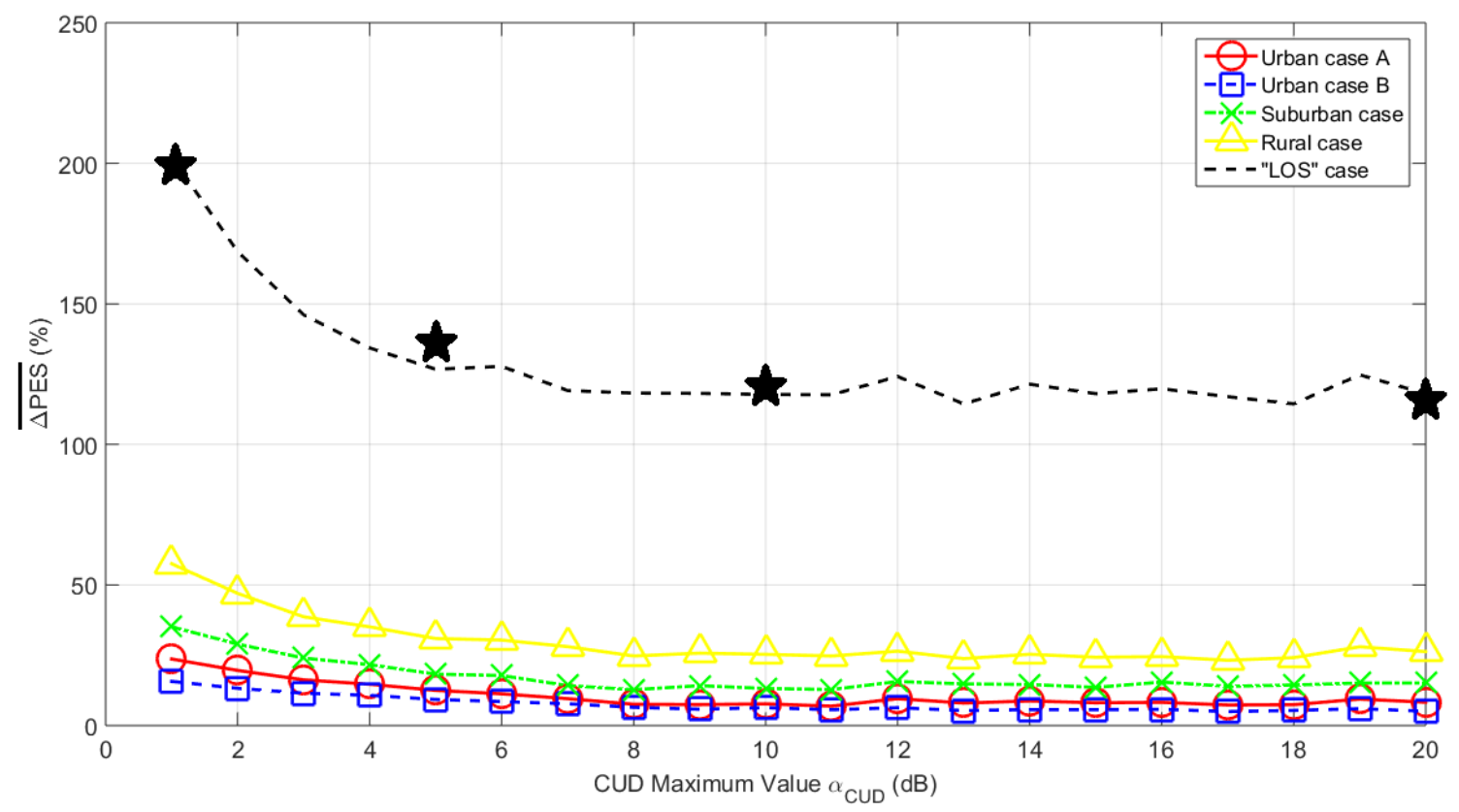

(a)

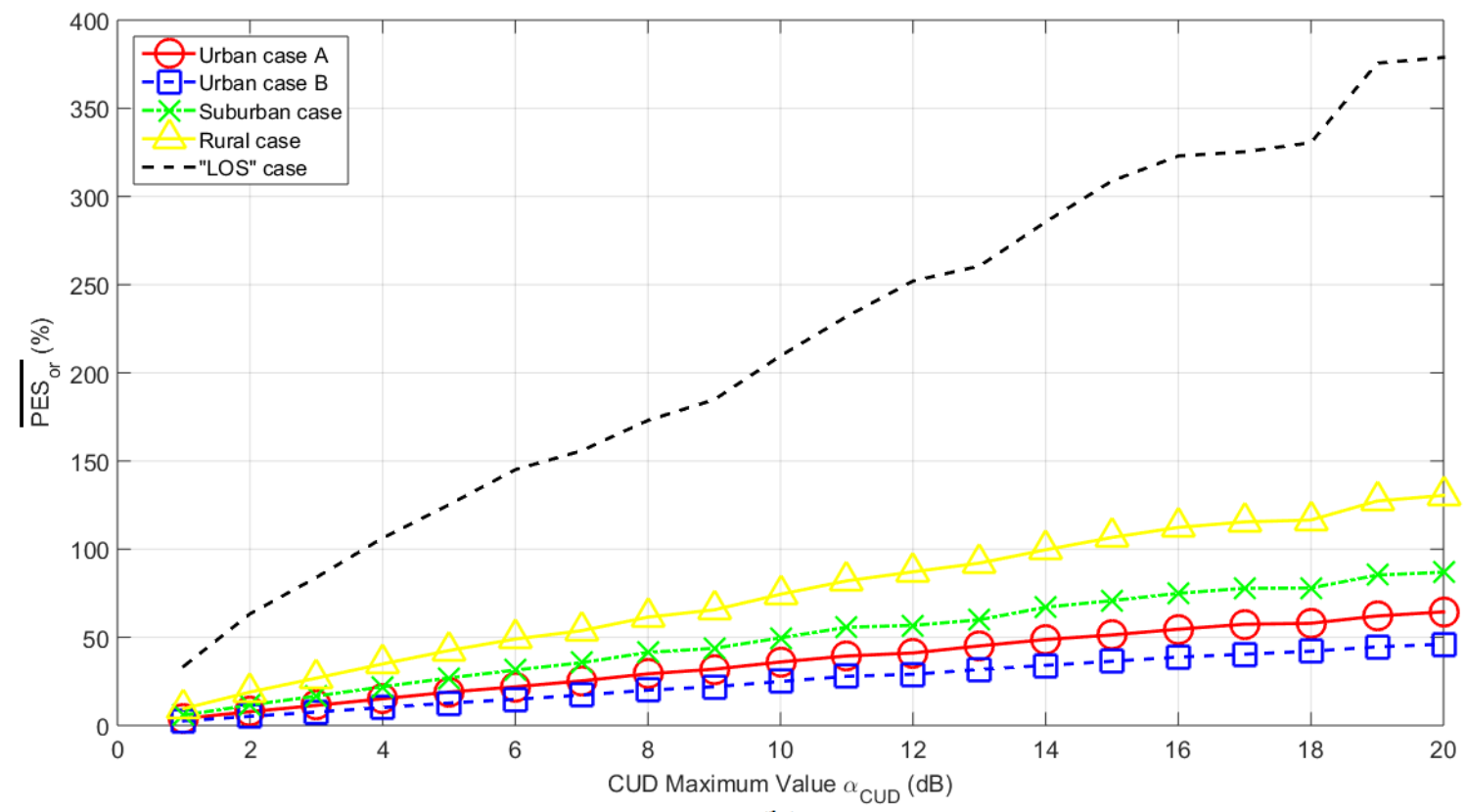

(b) 


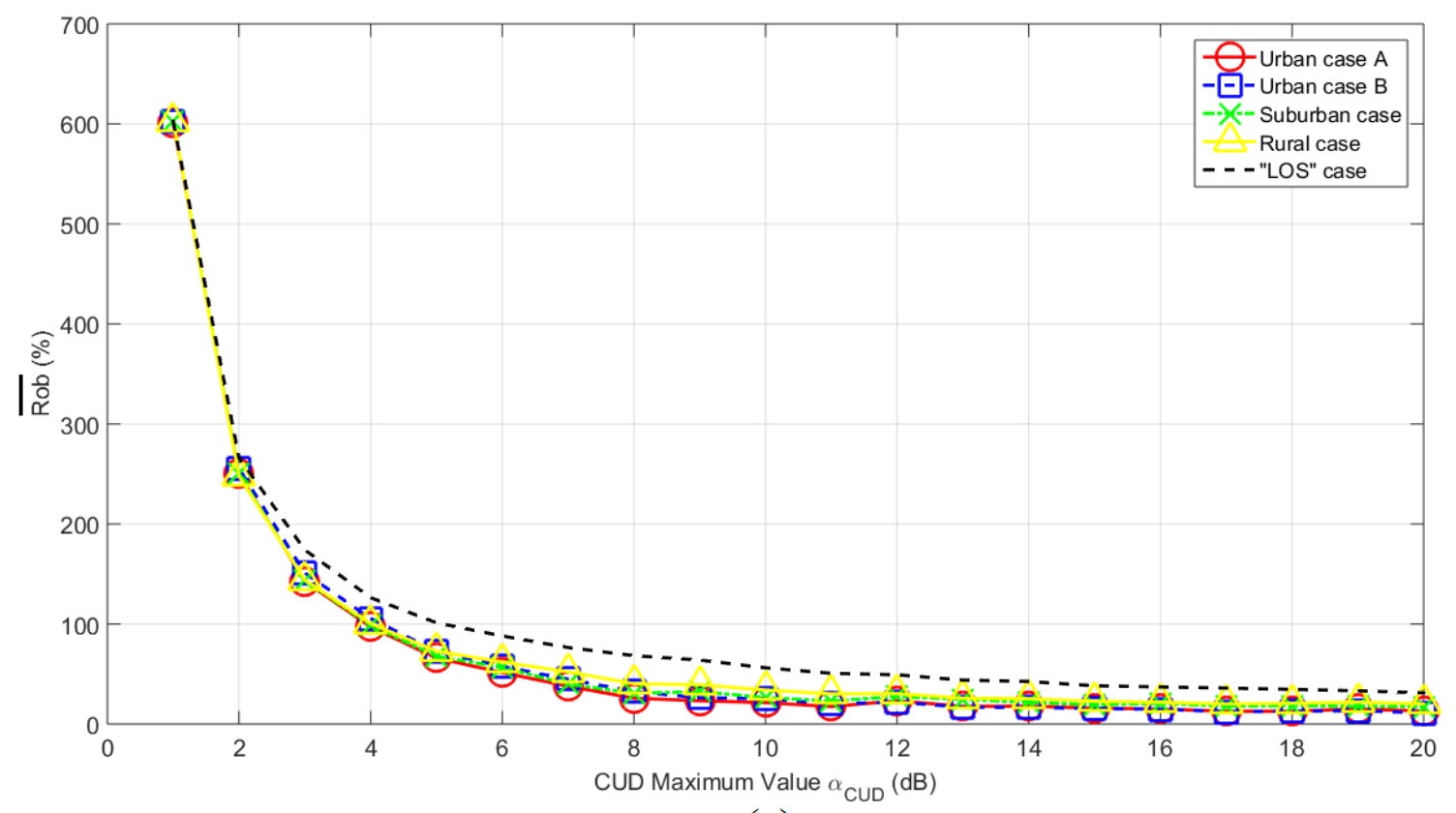

(c)

Fig. 7. PES submetrics of HS-DET method for the five original indicative OV LV BPL topologies of [1] when hook length of $5 \mathrm{~m}$, hook distance from the transmitting end of $300 \mathrm{~m}$ and matched hook termination are assumed for various maximum values $a_{\text {CUD }}$. (a) $\overline{\triangle P E S}$. (b) $\overline{P E S_{\text {or }}}$. (c) $\overline{R o b}$.

By comparing Figs. 1(a)-(c) with the respective Figs. 7(a)-(c), the three categories of OV LV BPL topologies concerning their behavior of $\overline{\triangle P E S}$ and $\overline{P E S_{\mathrm{rr}}}$ is also observed during the installation of smart hooks. Similarly to the open-circuit hooks, HSDET method can more easily detect the energy theft via smart hooks first in "LOS" case, second in good channel case and third in bad channel case. The only difference by comparing curves of given OV LV BPL topology is that $\overline{\triangle P E S}$ values of smart hooks are significantly lower than the respective ones of open-circuit short hooks.

By comparing Fig. 1(c) and Fig. 7(c), $\overline{R o b}$ curves of the examined OV LV BPL topologies almost coincide for given energy theft technique. But the $\overline{R o b}$ values of the smart hook technique are above the half of the respective ones of the open-circuit short length hooks. This result indicates the difficulty of HS-DET method to detect the energy theft when smart hooks can be applied.

As the comparison between smart hook technique and very long hook technique is regards, by comparing Figs. 2-6 with Fig. 7(a), it is obvious that $\overline{\triangle P E S}$ behavior of smart hooks present similarities with the behavior of very long hooks. For given OV LV BPL topology, $\overline{\triangle P E S}$ behavior of Fig. 7(a) that describes the smart hook technique can be safely approximated by the respective black stars $\overline{\triangle P E S}$ values of Figs. 2-6 that anyway describe the very long hook technique. Indeed, the black stars of Figs. 6(a)-(d), which describe the impact of very long hook technique on $\overline{\triangle P E S}$ of "LOS" case, have been transferred to Fig. 7(a) where the impact of smart hook technique on $\overline{\triangle P E S}$ of "LOS" case is also described. The almost coincidence of black stars and the black dashed line indicates the $\overline{\triangle P E S}$ agreement of these two energy theft techniques that is anyway expected due to their matched termination objective. 
In numerical terms and on the basis of the $\overline{\triangle P E S}$ and $\overline{R o b}$ thresholds of Sec.3.1, $\overline{\triangle P E S}$ and $\overline{R o b}$ values of the modified OV LV BPL topologies of Figs 7(a)-(c) are reported in Table 2 when maximum value $a_{\text {CUD }}$ of measurement differences is assumed to be equal to $20 \mathrm{~dB}$.

Table 2. $\overline{\triangle P E S}$ and $\overline{R o b}$ of Indicative OV LV BPL Topologies when $L_{\mathrm{bh}}=5 \mathrm{~m}$ and $D_{\mathrm{h}}=300 \mathrm{~m}$ (matched hook termination) for maximum values $a_{\mathrm{CUD}}$ equal to $20 \mathrm{~dB}$

\begin{tabular}{|c|c|c|c|c|c|}
\hline & \multicolumn{5}{|c|}{ Modified OV LV BPL Topology } \\
\hline & Urban case A & Urban case B & Suburban case & Rural case & "LOS" case \\
\hline $\begin{array}{c}\overline{\Delta P E S} \\
(\%)\end{array}$ & 8.29 & 5.09 & 15.11 & 26.30 & 118.08 \\
\hline $\begin{array}{c}\boldsymbol{R} \boldsymbol{\text { \%b }} \\
(\%)\end{array}$ & 12.86 & 11.04 & 17.39 & 20.16 & 31.17 \\
\hline
\end{tabular}

Comparing $\overline{\triangle P E S}$ values of Table 2 with the respective values of Table $1, \overline{\triangle P E S}$ and $\overline{R o b}$ values of smart hooks are almost the half in comparison with the respective ones of open-circuit hook of the same hook length in the OV LV BPL topologies of the "LOS" and good channel cases. $\overline{\triangle P E S}$ and $\overline{R o b}$ differences remain important even in the OV LV BPL topologies of the bad channel case. Anyway, in all the cases examined $\overline{\triangle P E S}$ values are always above $0 \%$ and, hence, HS-DET method can successfully detect the energy theft in all the cases. Again, the matched terminations are proven to be a challenge for the HS-DET method in comparison with the open-circuit terminations.

Already been mentioned in the case of the very long hooks, the detection of the energy theft by the HS-DET is accomplished through the strict $\overline{\triangle P E S}$ and $\overline{R o b}$ thresholds in the OV LV BPL topologies of "LOS" and good channel cases while the loose $\overline{\triangle P E S}$ and $\overline{R o b}$ thresholds need to be adopted for the OV LV BPL topologies of the bad channel case. In accordance with the analysis made in this paper, it is evident that the strict $\overline{\triangle P E S}$ and $\overline{R o b}$ thresholds guarantee a safer decision concerning the existence of a hook style energy theft in contrast with the loose $\overline{\triangle P E S}$ and $\overline{R o b}$ thresholds.

In addition, measurement differences influence the values of $\overline{\triangle P E S}$ even in smart hook case regardless of the examined OV LV BPL topology further deteriorating the quality of decisions regarding the existence of the energy theft. In fact, with reference to Fig. 7(a), when measurement differences are not so severe (i.e., the maximum value $a_{\text {CUD }}$ is below $6 \mathrm{~dB}$ ), the detection of the energy theft by HS-DET method can be made through the strict $\overline{\triangle P E S}$ threshold in all the OV LV BPL topologies examined.

Finally, it has been proven that the combined operation of smart hooks with high measurement differences can significantly complicate the decision of HS-DET method concerning the existence of energy theft but the implementation of this combined operation is not an easy task. From one side, the use of very sophisticated technology is required so that a smart hook can be implemented while from the other side the use of appropriate jammers that can create measurement differences of such maximum values is questionable. Anyway, even if the aforementioned prerequisites can be achieved, HSDET method can detect the energy theft even in the most challenging cases of the aggravated urban OV LV BPL topologies through the adoption of the loose $\overline{\triangle P E S}$ threshold. 
Concluding this paper and synopsizing the findings of this paper in relation with three special cases of [1], HS-DET method succeeds in detecting hook style energy thefts that may occur across the OV LV BPL networks even if high measurement differences occur and specialized jamming techniques, which focus on the exploitation of the matched terminations, are applied. In fact, through the definition of two types of $\overline{\triangle P E S}$ threshold (i.e., strict and loose $\overline{\triangle P E S}$ threshold), the quality of decision concerning the existence of a hook style energy theft can be evaluated. As the installation of very long hooks is examined in order to mask the energy theft, certain crucial disadvantages have been spotted, namely: (i) the quality of the power delivery; (ii) the apparent size of the energy theft equipment; (iii) the need for very long hooks (above $2000 \mathrm{~m}-5000 \mathrm{~m}$ ) so that the behavior of the hook can be appeared as hook with matched termination and create $\overline{\triangle P E S}$ values that are below the strict $\overline{\triangle P E S}$ threshold; and (iv) even if very high measurement differences can be produced intentionally, HS-DET method detects the energy theft through the loose $\overline{\triangle P E S}$ threshold. As the installation of smart hooks is examined in order to jam the HS-DET method, several disadvantages have been reported, namely: (i) the need for sophisticated technology so that a smart hook can be implemented; and (ii) even if very high measurement differences can be produced intentionally, HS-DET method detects the energy theft through the loose $\overline{\triangle P E S}$ threshold.

After the analysis of this paper, the future research consists of three sophisticated cases that need further investigation concerning the behavior of HS-DET method, namely: (i) The existence of different CUD measurement differences of the same maximum value $a_{\text {CUD }}$ in eqs. (2) and (3). This scenario examines the possibility of not recording the last measurement of $\overline{P E S_{\mathrm{nr}}}$ of eq. (2) and only $\overline{P E S_{\text {mnd }}}$ of eq. (3) is available at the moment of the hook installation; (ii) The installation of second smart hook in order to further jam HS-DET method. In this case, the first smart hook will perform the energy theft while the second smart hook will act as a mask; and (iii) The impact of the assumption of full interconnection during the computation. This issue has to do with the operation of the hybrid method and the assumption made concerning the connection to all three phases and the neutral. All the aforementioned three sophisticated aim at jamming HS-DET method while the hook style energy theft detection performance of the HS-DET method regarding these three cases is further investigated in [35].

\section{Conclusions}

This paper has focused on the performance of HS-DET method when the three special cases of [1] are addressed. As the first special case is examined, there is no threshold of CUD maximum values $a_{\text {CUD }}$ of measurement differences below $20 \mathrm{~dB}$ that HS-DET method could not detect the hook style energy theft. Even if high CUD measurement differences occur, HS-DET method can detect the energy theft through its strict $\overline{\triangle P E S}$ threshold of $10 \%$ in the vast majority of the OV LV BPL topologies examined while the loose $\overline{\triangle P E S}$ threshold of HS-DET method of $0 \%$ allows the energy theft in the remaining OV LV BPL topologies (e.g., urban OV LV BPL topologies when very high measurement differences occur). As the second special case is examined, the installation of very long hooks in order to mask the hook existence during the application of HS-DET method can indeed make the energy theft detection less easy by HS-DET 
method but again in all the cases HS-DET method through its strict and loose $\overline{\triangle P E S}$ thresholds. The $\overline{\triangle P E S}$ behavior of very long hooks (i.e., hook lengths above $5000 \mathrm{~m}$ ) resembles to the matched termination $\overline{\triangle P E S}$ behavior. However, the main disadvantage of this energy theft technique is its practicability that is questionable due to its size. As the third special case is examined, the $\overline{\triangle P E S}$ behavior of "smart" hooks resembles to the respective behavior of very long hooks. Although this energy theft technique imposes difficulties to HS-DET method especially when these "smart" hooks are installed in urban OV LV BPL topologies, in all the cases HS-DET method through its strict and loose $\overline{\triangle P E S}$ thresholds can detect the energy theft. The main disadvantage of the "smart" hook energy theft technique is its sophisticated technology. In order to evaluate HS-DET method during extreme scenarios of hook style energy theft, three questions need further examination and future research: (i) The existence of different CUD measurement differences of the same maximum value $a_{\text {CUD }}$ during the last transfer function recording and the hook insertion moment; (ii) The installation of second smart hook in order to mask the presence of the first one; and (iii) The impact of the assumption of full interconnection during the computations by HS-DET method.

\section{Appendix - Can High Measurement Differences Trigger the Hook Style Energy Theft Alarm of HS-DET Method}

The impact of measurement differences on $\overline{\triangle P E S}, \overline{P E S_{\mathrm{nr}}}$ and $\overline{R o b}$ of the original indicative OV LV BPL topologies is examined in this Appendix. For this purpose, during the computation of the aforementioned PES submetrics from eqs (2)-(5), the modified measured coupling scheme channel transfer function $\overline{H_{\text {mnd }}^{\mathrm{C}}}\{\cdot\}$ is assumed to be equal to the original measured coupling scheme channel transfer function $\overline{H_{\mathrm{n}}^{\mathrm{C}}}\{\cdot\}$. With reference to eqs. (2) and (3), $\overline{P E S_{\text {man }}}$ becomes equal to $\overline{P E S_{\mathrm{or}}}$ when the aforementioned assumption occurs. Taking under consideration the last observation, the following conclusions can be deduced concerning $\overline{\triangle P E S}, \overline{P E S_{\mathrm{rr}}}$ and $\overline{R o b}$, say:

- $\overline{\triangle P E S}$ : With reference to eq. (4), $\overline{\triangle P E S}$ becomes equal to zero. Therefore, either the loose $\overline{\triangle P E S}$ threshold (i.e., $\overline{\triangle P E S}$ above $0 \%$ ) or the strict $\overline{\triangle P E S}$ threshold (i.e., $\overline{\triangle P E S}$ above $10 \%$ ) cannot be exceeded by the measurement differences.

- $\overline{P E S_{\mathrm{nr}}}$ : With reference to eq. (2), $\overline{P E S_{\mathrm{nr}}}$ is independent of the modified measured coupling scheme channel transfer function since it depends only on the original measured and original theoretical coupling scheme channel transfer function. $\overline{P E S_{\mathrm{nr}}}$ is already plotted in Fig. 1(b).

- $\overline{R o b}$ : With reference to eq. (5), $\overline{R o b}$ is equal to $0 \%$ since $\overline{\triangle P E S}$ is equal to $0 \%$. Again, either the loose $\overline{R o b}$ threshold (i.e., $\overline{R o b}$ above $0 \%$ ) or the strict $\overline{R o b}$ threshold (i.e., $\overline{R o b}$ above $20 \%$ ) cannot be exceeded by the measurement differences.

Concluding this Appendix, when there is no hook style energy theft the measurement differences cannot trigger the energy theft alarm. This result is an important safety characteristic of the HS-DET method. 


\section{CONFLICTS OF INTEREST}

The author declares that there is no conflict of interests regarding the publication of this paper.

\section{References}

[1] A. G. Lazaropoulos, "Detection of Energy Theft in Overhead Low-Voltage Power Grids - The Hook Style Energy Theft in the Smart Grid Era," Trends in Renewable Energy, vol. 5, no. 1, pp. 12 - 46, Oct. 2018. [Online]. Available: http://futureenergysp.com/index.php/tre/article/view/81/pdf

[2] A. G. Lazaropoulos, "Measurement Differences, Faults and Instabilities in Intelligent Energy Systems - Part 1: Identification of Overhead High-Voltage Broadband over Power Lines Network Topologies by Applying Topology Identification Methodology (TIM)," Trends in Renewable Energy, vol. 2, no. 3, pp. $85-112$, Oct. $2016 . \quad$ [Online]. Available: http://futureenergysp.com/index.php/tre/article/download/26/32

[3] A. G. Lazaropoulos, "Measurement Differences, Faults and Instabilities in Intelligent Energy Systems - Part 2: Fault and Instability Prediction in Overhead High-Voltage Broadband over Power Lines Networks by Applying Fault and Instability Identification Methodology (FIIM)," Trends in Renewable Energy, vol. 2, no. 3, pp. 113 - 142, Oct. 2016. [Online]. Available: http://futureenergysp.com/index.php/tre/article/view/27/33

[4] A. G. Lazaropoulos, "Power Systems Stability through Piecewise Monotonic Data Approximations - Part 2: Adaptive Number of Monotonic Sections and Performance of L1PMA, L2WPMA and L2CXCV in Overhead Medium-Voltage Broadband over Power Lines Networks," Trends in Renewable Energy, vol. 3, no. 1, pp. $33-60$, Jan. 2017. [Online]. Available: http://futureenergysp.com/index.php/tre/article/view/30/35

[5] A. G. Lazaropoulos, "Power Systems Stability through Piecewise Monotonic Data Approximations - Part 1: Comparative Benchmarking of L1PMA, L2WPMA and L2CXCV in Overhead Medium-Voltage Broadband over Power Lines Networks," Trends in Renewable Energy, vol. 3, no. 1, pp. 2 - 32, Jan. 2017. [Online]. Available: http://futureenergysp.com/index.php/tre/article/view/29/34

[6] A. G. Lazaropoulos, "Main Line Fault Localization Methodology in Smart Grid Part 1: Extended TM2 Method for the Overhead Medium-Voltage Broadband over Power Lines Networks Case," Trends in Renewable Energy, vol. 3, no. 3, pp. 2-25, Dec. $2017 . \quad$ [Online]. Available: http://futureenergysp.com/index.php/tre/article/view/36

[7] A. G. Lazaropoulos, "Main Line Fault Localization Methodology in Smart Grid Part 2: Extended TM2 Method, Measurement Differences and L1 Piecewise Monotonic Data Approximation for the Overhead Medium-Voltage Broadband over Power Lines Networks Case," Trends in Renewable Energy, vol. 3, no. 3, pp. 26-61, Dec. 2017. [Online]. Available: http://futureenergysp.com/index.php/tre/article/view/37

[8] A. G. Lazaropoulos, "Main Line Fault Localization Methodology in Smart Grid Part 3: Main Line Fault Localization Methodology (MLFLM)," Trends in 
Renewable Energy, vol. 3, no. 3, pp. 62-81, Dec. 2017. [Online]. Available: http://futureenergysp.com/index.php/tre/article/view/38

[9] A. G. Lazaropoulos, "Towards Modal Integration of Overhead and Underground Low-Voltage and Medium-Voltage Power Line Communication Channels in the Smart Grid Landscape: Model Expansion, Broadband Signal Transmission Characteristics, and Statistical Performance Metrics (Invited Paper)," ISRN Signal Processing, vol. 2012, Article ID 121628, pp. 1-17, 2012. [Online]. Available: http://www.hindawi.com/isrn/sp/2012/121628/

[10] A. G. Lazaropoulos, "Towards broadband over power lines systems integration: Transmission characteristics of underground low-voltage distribution power lines," Progress in Electromagnetics Research B, 39, pp. 89-114, 2012. [Online]. Available: http://www.jpier.org/PIERB/pierb39/05.12012409.pdf

[11] A. G. Lazaropoulos and P. G. Cottis, "Transmission characteristics of overhead medium voltage power line communication channels," IEEE Trans. Power Del., vol. 24, no. 3, pp. 1164-1173, Jul. 2009.

[12] A. G. Lazaropoulos and P. G. Cottis, "Capacity of overhead medium voltage power line communication channels," IEEE Trans. Power Del., vol. 25, no. 2, pp. 723733, Apr. 2010.

[13] A. G. Lazaropoulos and P. G. Cottis, "Broadband transmission via underground medium-voltage power lines-Part I: transmission characteristics," IEEE Trans. Power Del., vol. 25, no. 4, pp. 2414-2424, Oct. 2010.

[14] A. G. Lazaropoulos and P. G. Cottis, "Broadband transmission via underground medium-voltage power lines-Part II: capacity," IEEE Trans. Power Del., vol. 25, no. 4, pp. 2425-2434, Oct. 2010.

[15] A. G. Lazaropoulos, "Broadband transmission characteristics of overhead highvoltage power line communication channels," Progress in Electromagnetics Research B, vol. 36, pp. 373-398, 2012. [Online]. Available: http://www.jpier.org/PIERB/pierb36/19.11091408.pdf

[16] A. G. Lazaropoulos, "Green Overhead and Underground Multiple-Input MultipleOutput Medium Voltage Broadband over Power Lines Networks: Energy-Efficient Power Control," Springer Journal of Global Optimization, vol. 2012 / Print ISSN 0925-5001, pp. 1-28, Oct. 2012.

[17] A. G. Lazaropoulos, "Deployment Concepts for Overhead High Voltage Broadband over Power Lines Connections with Two-Hop Repeater System: Capacity Countermeasures against Aggravated Topologies and High Noise Environments," Progress in Electromagnetics Research B, vol. 44, pp. 283-307, 2012. [Online]. Available: http://www.jpier.org/PIERB/pierb44/13.12081104.pdf

[18] A. G. Lazaropoulos, "Broadband transmission and statistical performance properties of overhead high-voltage transmission networks," Hindawi Journal of Computer Networks and Commun., 2012, article ID 875632, 2012. [Online]. Available: http://www.hindawi.com/journals/jcnc/aip/875632/

[19] P. Amirshahi and M. Kavehrad, "High-frequency characteristics of overhead multiconductor power lines for broadband communications," IEEE J. Sel. Areas Commun., vol. 24, no. 7, pp. 1292-1303, Jul. 2006.

[20] T. Calliacoudas and F. Issa, "“Multiconductor transmission lines and cables solver," An efficient simulation tool for plc channel networks development," presented at the IEEE Int. Conf. Power Line Communications and Its Applications, Athens, Greece, Mar. 2002. 
[21] T. Sartenaer and P. Delogne, "Deterministic modelling of the (Shielded) outdoor powerline channel based on the multiconductor transmission line equations," IEEE J. Sel. Areas Commun., vol. 24, no. 7, pp. 1277-1291, Jul. 2006.

[22] C. R. Paul, Analysis of Multiconductor Transmission Lines. New York: Wiley, 1994.

[23] H. Meng, S. Chen, Y. L. Guan, C. L. Law, P. L. So, E. Gunawan, and T. T. Lie, "Modeling of transfer characteristics for the broadband power line communication channel," IEEE Trans. Power Del., vol. 19, no. 3, pp. 1057-1064, Jul. 2004.

[24] B. Li, D. Mansson, and G. Yang, "An efficient method for solving frequency responses of power-line networks," Progress in Electromagnetics Research B, Vol. 62, 303-317, 2015. DOI: $10.2528 /$ PIERB15013008 http://www.jpier.org/pierb/pier.php?paper $=15013008$

[25] M. Chaaban, K. El KhamlichiDrissi, and D. Poljak, "Analytical model for electromagnetic radiation by bare-wire structures," Progress in Electromagnetics Research B, Vol. 45, 395-413, 2012. DOI:10.2528/PIERB12091102 http://www.jpier.org/pierb/pier.php?paper $=12091102$

[26] Y. H. Kim, S. Choi, S. C. Kim, and J. H. Lee, "Capacity of OFDM two-hop relaying systems for medium-voltage power-line access networks," IEEE Trans. Power Del., vol. 27, no. 2, pp. 886-894, Apr. 2012.

[27] A. G. Lazaropoulos, "Best L1 Piecewise Monotonic Data Approximation in Overhead and Underground Medium-Voltage and Low-Voltage Broadband over Power Lines Networks: Theoretical and Practical Transfer Function Determination," Hindawi Journal of Computational Engineering, vol. 2016, Article ID 6762390, 24 pages, 2016. DOI:10.1155/2016/6762390. [Online]. Available: https://www.hindawi.com/journals/jcengi/2016/6762390/cta/

[28] A. G. Lazaropoulos, "New Coupling Schemes for Distribution Broadband over Power Lines (BPL) Networks," Progress in Electromagnetics Research B, vol. 71, pp. 39-54, 2016. [Online]. Available: http://www.jpier.org/PIERB/pierb71/02.16081503.pdf

[29] A. G. Lazaropoulos, "Broadband Performance Metrics and Regression Approximations of the New Coupling Schemes for Distribution Broadband over Power Lines (BPL) Networks," Trends in Renewable Energy, vol. 4, no. 1, pp. 43 73, 2018. [Online]. Available: http://www.futureenergysp.com/index.php/tre/article/view/59/pdf

[30] OPERA1, D44: Report presenting the architecture of plc system, the electricity network topologies, the operating modes and the equipment over which PLC access system will be installed, IST Integr. Project No 507667, Dec. 2005.

[31] OPERA1, D5: Pathloss as a function of frequency, distance and network topology for various LV and MV European powerline networks. IST Integrated Project No 507667, Apr. 2005.

[32] A. G. Lazaropoulos, "Factors Influencing Broadband Transmission Characteristics of Underground Low-Voltage Distribution Networks," IET Commun., vol. 6, no. 17, pp. 2886-2893, Nov. 2012.

[33] J. Anatory, N. Theethayi, and R. Thottappillil, "Power-line communication channel model for interconnected networks-Part II: Multiconductor system," IEEE Trans. Power Del., vol. 24, no. 1, pp. 124-128, Jan. 2009.

[34] J. Anatory, N. Theethayi, R. Thottappillil, M. M. Kissaka, and N. H. Mvungi, "The effects of load impedance, line length, and branches in typical low-voltage channels 
of the BPLC systems of developing countries: transmission-line analyses," IEEE Trans. Power Del., vol. 24, no. 2, pp. 621-629, Apr. 2009.

[35] A. G. Lazaropoulos, "Special Cases during the Detection of the Hook Style Energy Theft in Overhead Low-Voltage Power Grids through HS-DET Method - Part 2: Different Measurement Differences, Feint "Smart" Hooks and Hook Interconnection Issues," Trends in Renewable Energy, DOI: 10.17737/tre.2019.5.1.0083.

Article copyright: (C) 2019 Athanasios G. Lazaropoulos. This is an open access article distributed under the terms of the Creative Commons Attribution 4.0 International License, which permits unrestricted use and distribution provided the original author and source are credited. 